
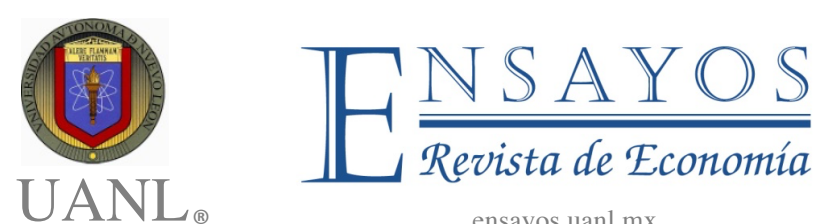

ensayos.uanl.mx

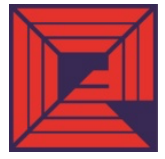

Facultad de Economía

\title{
Efectos de la gran recesión sobre la distribución del ingreso en México
}

\section{The Effects of the Great Recession on the Income Distribution in Mexico}

\section{María del Rosario Ruiz Hernández * Leonardo Adalberto Gatica*}

\begin{tabular}{|c|c|}
\hline $\begin{array}{l}\text { Información del } \\
\text { artículo }\end{array}$ & Resumen \\
\hline Recibido: & \multirow{8}{*}{$\begin{array}{l}\text { En este artículo analizamos el impacto que la Gran } \\
\text { Recesión tuvo sobre la desigualdad del ingreso en México } \\
\text { Estudiamos tres diferentes dimensiones del ingreso de los } \\
\text { hogares: el ingreso por trabajo asalariado, el ingreso tota } \\
\text { por trabajo y el ingreso monetario. Encontramos que e } \\
\text { impacto negativo de la contracción económica sobre la } \\
\text { inequidad del ingreso monetario no se observa para e } \\
\text { ingreso por trabajo asalariado. Esta diferencia se explica } \\
\text { por la caída en el ingreso por negocios que afectó en mayor } \\
\text { proporción a los hogares del decil más alto, que a los } \\
\text { hogares en los cuatro deciles más bajos de la distribución } \\
\text { Esta caída desproporcionada, de los ingresos por negocios } \\
\text { redujo ligeramente la desigualdad de los ingresos totales } \\
\text { del trabajo. En el nivel regional se observan efectos } \\
\text { heterogéneos; algunos de ellos se contraponen a lo } \\
\text { observado en promedio, en el nivel nacional. }\end{array}$} \\
\hline 20 abril 2020 & \\
\hline $\begin{array}{l}\text { Aceptado: } \\
17 \text { febrero } 2021\end{array}$ & \\
\hline $\begin{array}{l}\text { Clasificación JEL: } \\
\text { D30; D31; O11 }\end{array}$ & \\
\hline Palabras clave: & \\
\hline México; Gran & \\
\hline $\begin{array}{l}\text { Recesión; Distribución } \\
\text { del Ingreso; }\end{array}$ & \\
\hline Desigualdad & \\
\hline
\end{tabular}

* Universidad de Guadalajara, rosario.ruiz.h@hotmail.com; ${ }^{* *}$ Universidad de Guadalajara, leonardo.gatic@gmal.com

ISSN Electrónico: 2448-8402 | ISSN Impreso: 1870-221X | (C2021 Los autores @ (c) 


\begin{tabular}{|c|c|}
\hline Article information & Abstract \\
\hline $\begin{array}{l}\text { Received: } \\
20 \text { April } 2020\end{array}$ & $\begin{array}{l}\text { In this paper, we explore the impact of the Great Recession } \\
\text { on income inequality in Mexico. We analyze three } \\
\text { different dimensions of household income: wages and }\end{array}$ \\
\hline $\begin{array}{l}\text { Accepted: } \\
17 \text { February } 2021\end{array}$ & $\begin{array}{l}\text { salaries, earnings, and monetary income. We found that } \\
\text { the negative effect of the downturn on household income }\end{array}$ \\
\hline $\begin{array}{l}\text { JEL Classification: } \\
\text { D30; D31; O11 }\end{array}$ & $\begin{array}{l}\text { inequality is mainly observed in monetary income, but not } \\
\text { for wages and salaries. This divergence is explained by the } \\
\text { significant contraction of business income that }\end{array}$ \\
\hline $\begin{array}{l}\text { Keywords: } \\
\text { Mexico; Great } \\
\text { Recession; Income } \\
\text { distribution; Inequality }\end{array}$ & $\begin{array}{l}\text { households compared to the bottom } 40 \text { percent. Business } \\
\text { income losses slightly reduced earnings inequality. We } \\
\text { also found heterogeneous regional effects, some of them } \\
\text { opposed to the national average. }\end{array}$ \\
\hline
\end{tabular}

\section{Introducción}

Dentro de los análisis sobre la distribución del ingreso, uno de los puntos que ha llamado más la atención, es el efecto que el ciclo económico y las crisis tienen sobre la desigualdad (Parker, 1998; Maliar, Maliar, y Mora, 2005; Piketty y Saez, 2003; Piketty, 2014; Piketty y Zucman, 2014). En los últimos años, varios trabajos se han enfocado en los efectos que la crisis de 2008 y la Gran Recesión han tenido sobre la distribución del ingreso en distintos países (Jenkins et al., 2013-B; Piketty y Saez, 2013; Pfeffer et al., 2013; Camacho y Palmieri, 2019; Brzezinski, 2018). En este trabajo, buscamos analizar y describir el impacto del choque negativo que significó la Gran Recesión sobre los principales componentes diferentes del ingreso de los hogares, y las consecuencias sobre su distribución en el nivel macroeconómico, para el caso de México. Analizamos diferentes componentes del ingreso total de los hogares provenientes de distintas fuentes, las cuales incluyen: salarios, ingresos por negocios, rentas producto de sus ahorros o inversiones, y de transferencias que reciben de otros hogares o del gobierno. De igual forma, analizamos el impacto de la crisis en diferentes regiones del país que, dadas las diferencias en su estructura económica, pudieran haber respondido de manera distinta a este choque negativo.

La Gran Recesión que inició con la crisis de 2007 causó una contracción en la economía global que no se había vivido desde la Gran Depresión de 1929 (Grabka, 2015). En 2008, el PIB del mundo era de 64,413 billones de dólares; para el 2009, había disminuido en más de mil billones de dólares. Países como Alemania, Argentina, Japón, Italia y México perdieron más del $5 \%$ de su producto interno bruto entre 2008 y $2009^{1}$. Diferentes trabajos muestran que este evento tuvo consecuencias importantes en la distribución del ingreso en distintos países. En general, como consecuencia de la contracción económica,

1 Datos del Banco Mundial. 
se observó una caída en el ingreso y en la riqueza de los diferentes grupos socioeconómicos, aunque se argumenta que los hogares menos favorecidos son quienes sufrieron un mayor impacto negativo, y han tenido menos posibilidades de recuperarse una vez pasada la crisis (Wolf 2013; Pfeffer et al., 2013; Piketty y Saez, 2013). Gasparini y Lustig (2011) documentaron las consecuencias de diferentes crisis en Brasil, Argentina y México, mostrando que los aumentos en la desigualdad en estos países se relacionan con los periodos de crisis por los cuales atravesaron. Sin embargo, también se ha observado una tendencia descendente en los indicadores de desigualdad para América Latina, durante la primera década del siglo XXI (Gasparini y Lustig, 2011; López-Calva y Lustig, 2010; López Calva, Lustig y Ortiz-Juárez, 2013); en el caso de México, López Calva, Lustig y Ortiz-Juárez (2013) documentan un ligero aumento en la desigualdad de 2006 a 2008, seguido de un descenso para el 2010.

Nuestro análisis utiliza datos de la Encuesta Nacional de Ingreso Gasto de los Hogares (ENIGH), desde 2006 hasta 2018. En contraste con trabajos previos, consideramos diferentes dimensiones del ingreso con lo que podemos tener una mejor idea del efecto que tuvo la contracción económica causada por la Gran Recesión en México, sobre distintas fuentes de ingreso de los hogares. Para nuestro análisis, utilizamos tres dimensiones de ingreso: Ingreso Salarial (IS) que incluye únicamente los ingresos que reportan los hogares por sueldos y salarios y sus diferentes componentes (trabajo subordinado); Ingreso Total del Trabajo (ITT) en el que incluimos además del ingreso salarial, el ingreso proveniente de negocios (trabajo subordinado e independiente y por autoempleo); y por último, Ingreso Monetario (IM), el cual además de los anteriores, incluye rentas y transferencias.

En nuestro estudio encontramos un patrón similar al que reportan trabajos previos, sobre la desigualdad del ingreso corriente, en el que se observa una caída en la desigualdad entre 2008 y 2010 (López Calva, Lustig y Ortiz-Juárez, 2013; Cortés y Vargas, 2017). En nuestro caso, encontramos también una caída en la desigualdad del ingreso monetario, que es la dimensión más cercana al ingreso corriente que utilizan estudios previos. Sin embargo, para el caso del ingreso salarial y el ingreso por trabajo total, el comportamiento es distinto. La desigualdad del ingreso por trabajo total, al igual que el ingreso monetario, tuvo un descenso en 2010, aunque mucho menor que el ingreso monetario. En el caso de los ingresos que provienen del trabajo asalariado, la desigualdad prácticamente permaneció estable de 2006 a 2008, a partir de ese año, inició un aumento hasta el 2012. Así, aún cuando durante la Gran Recesión podemos observar una caída en la desigualdad en el ingreso monetario total, encontramos que hubo un incremento en la desigualdad en los ingresos provenientes del trabajo asalariado, que está ligado al sector formal de la economía. La evidencia sugiere que la disminución de la desigualdad que se 
aprecia en el ingreso total por trabajo se debe principalmente al papel del ingreso por negocios propios y auto empleo, y que las transferencias reforzaron esa disminución en el ingreso monetario. Si bien la contracción económica durante el periodo de la Gran Recesión tuvo en promedio un impacto negativo en el ingreso, los hogares de todos los estratos socioeconómicos del país, los impactos relativos entre los diferentes grupos de hogares fueron muy distintos en las tres dimensiones de ingreso. La disminución de los indicadores de desigualdad en el ingreso monetario observado y que también es reportado por trabajos anteriores, es consecuencia de una caída más profunda en el ingreso monetario de los hogares que se ubican los deciles altos de la distribución, en comparación con los hogares con ingresos bajos, relacionado con las transferencias gubernamentales y la caída en las rentas y los ingresos por negocios. Sin embargo, la diferencia de esos impactos es mucho menor para el ingreso total por trabajo y se revierten en el caso del ingreso por trabajo asalariado. Este comportamiento en el nivel nacional no necesariamente se replica en nivel regional. Al analizar la dinámica de las tres dimensiones de ingreso en ocho regiones, se observan patrones contrastantes fundamentalmente en el ingreso por trabajo asalariado y en el ingreso total por trabajo, así como el papel que las transferencias, aunque no necesariamente gubernamentales, juegan para generar un comportamiento más homogéneo en el caso del ingreso monetario.

El resto de este artículo se organiza de la siguiente manera. A continuación, discutimos la literatura que se ha enfocado en los efectos que las crisis tienen sobre la distribución del ingreso, y en particular aquella que se refiere a las consecuencias que la Gran Recesión tuvo en diversos países y en México. Posteriormente, describimos los datos que empleamos en el estudio y definimos las categorías de ingreso que conforman cada una de las dimensiones que construimos. En los siguientes dos apartados, presentamos los resultados y la evidencia que el análisis empírico muestra sobre las consecuencias de la Gran Recesión en la distribución de ingreso en México; primero, en el nivel nacional y luego, en el nivel regional. Finalmente, cerramos el articulo con las conclusiones.

\section{Los efectos de las crisis en la distribución del ingreso}

La relación entre la distribución del ingreso y los ciclos económicos es uno de los temas que más ha atraído la atención en el análisis económico (ver por ejemplo Parker (1998), para una revisión de la literatura); y en la última década, ha habido un importante desarrollo en la literatura enfocada en la relación de las crisis y la inequidad (Parker y Vissing-Jorgensen, 2009, 2010; Stiglitz 2009; Morelli y Atkinson 2015; Acemoglu, 2011; Piketty y Saez, 2013). Si bien sabemos que las crisis impactan, al menos en el corto plazo, la distribución del ingreso, existe también un conceso sobre la dificultad para 
identificar el efecto directo de las crisis sobre la distribución, debido a las respuestas institucionales y de política económica que se implementan durante estos eventos. A partir de Atkinson y Brandolini (2006), quienes con el objetivo de determinar los impactos que la globalización tiene sobre la desigualdad, se considera la prima salarial de trabajadores calificados, la proporción de trabajadores desempleados y trabajadores no calificados que sufren desempleo, así como la tasa impositiva sobre las ganancias del capital, Jenkins, Brandolini, Micklewright y Nolan (2012) retoman su metodología para analizar esas mismas variables, y estudiar el impacto de las crisis. Jenkins et al. (2012), muestran que el coeficiente de Gini es una función de diversas variables macroeconómicas, además de parámetros institucionales que modifican el estado de bienestar, por lo que es difícil aislar los efectos de las crisis sobre la distribución. De igual manera, algunos trabajos de Piketty y Saez (2003 y 2013) para Estados Unidos, muestran que la concentración del ingreso en el Top_10 disminuye en los periodos posteriores a las recesiones, señalan que la disminución se debe en gran medida a los cambios institucionales y de política fiscal, y no a los efectos de las crisis per se.

Dada la dificultad para identificar los impactos específicos de las contracciones sobre la desigualdad, en general, los análisis se han centrado en la evaluación del comportamiento del ingreso durante los periodos de recesión y crisis, sin que necesariamente se busque aislar el efecto de la contracción económica, de las respuestas de política. En nuestro estudio, si bien tampoco existen los datos para poder aislar el efecto de la crisis y separarlo del impacto de la política impositiva, y principalmente del gasto público redistributivo, que va más allá de las transferencias directas a los hogares y que pueden tener impactos en la desigualdad (Carmona y Camaal, 2018), sí hemos podido al menos aislar los efectos de las transferencias gubernamentales directas, que reciben las familias y que para el caso de México son probablemente uno de los principales canales de la política redistributiva, para disminuir tanto la pobreza como la desigualdad.

En la literatura no se ha identificado un patrón específico de los efectos que las crisis tienen sobre la desigualdad, y se tiende a concluir que el proceso redistributivo durante las crisis y en los periodos posteriores dependen de variables como la tendencia previa de la desigualdad, el tipo de crisis y sus causas, así como de factores institucionales.

Barlevy y Tsiddon (2006), por ejemplo, construyen un modelo en el que descomponen la desigualdad en un factor cíclico y un componente tendencial, y concluyen que el impacto de una crisis depende de la tendencia que tenga la desigualdad en el momento del choque: cuando la tendencia de la desigualdad en el largo plazo aumenta, la recesión incrementa la desigualdad, caso contrario cuando la tendencia es decreciente. La tendencia de la desigualdad 
está determinada por factores estructurales y muy probablemente también está asociada con la estructura institucional, por lo que podríamos esperar que los hallazgos de Barlevy y Tsiddon (2006) estuvieran relacionados con variables institucionales y de política fiscal.

Algunos trabajos, como por ejemplo Maliar, Maliar y Mora (2005) que utilizan un modelo de crecimiento con agentes heterogéneos para analizar el caso de los Estados Unidos, identificaron un comportamiento contracíclico de la desigualdad. Consistente con este resultado, Dimelis y Livada (1999) también han identificado un comportamiento contracíclico tanto para los Estados Unidos como para el Reino Unido; sin embargo, observan que en Grecia e Italia la desigualdad tiene un patrón mixto. En contraste con Maliar et al. (2005), Muriel y Sibieta (2009) analizan el caso de las últimas tres crisis en el Reino Unido, y concluyen que los efectos sobre la distribución dependen de las características de cada crisis y de los impactos diferenciados que pueda tener sobre distintos grupos socioeconómicos. Aunque estos últimos autores limitan sus conclusiones al Reino Unido, es muy posible que este sea, en general, el caso dado, ya que los efectos macroeconómicos dependen del tipo de crisis (Reinhart y Rogoff, 2011), y la transmisión de estos efectos sobre los diferentes grupos sociales, dependen del entramado institucional particular de cada economía. Eso implica que en países con regiones heterogéneas en sus dinámicas económicas y en su estructura socioeconómica, los efectos de las crisis en las regiones pueden ser contrastantes, como lo mostramos en nuestro estudio.

La heterogeneidad de la respuesta de la desigualdad frente a las recesiones se ha documentado más recientemente por Camacho y Palmieri (2019), quienes analizaron el impacto de los periodos de recesión sobre la desigualdad, utilizando una muestra de 43 países para el periodo de 1960 a 2016. Sus resultados muestran que las contracciones económicas, no necesariamente incrementan la desigualdad del ingreso, medida por el índice de Gini. Encuentran que, dentro de los tres años posteriores a la contracción, las recesiones tienden a disminuir la desigualdad de forma estadísticamente significativa en el $22 \%$ de los países estudiados; mientras únicamente en el $17 \%$ de los casos, la desigualdad aumenta. De acuerdo con sus resultados, si se consideran tanto los efectos significativos como los que no lo son, las recesiones tienden a disminuir la desigualdad en el 54\% del total de los países estudiados, mientras que en el resto la tienden a aumentar. Al considerar únicamente los países de la OCDE y los países de ingresos altos, los resultados generales se revierten y se observa que las recesiones tienden a tener un impacto positivo sobre la desigualdad. Ese también es el efecto que encuentran 
en el caso de México. ${ }^{2}$ Aún cuando los autores no controlan por el tipo de crisis o recesiones, y lo que se reporta es el promedio de los cambios en el índice de Gini, estos resultados llaman la atención, ya que los países más desarrollados cuentan con marcos institucionales y respuestas de política que esperaríamos disminuyeran el impacto de las crisis sobre la inequidad, como se plantea en otras investigaciones (Piketty y Saez, 2003 y 2013; Grabka, 2015; Muriel y Sibieta, 2009).

Para el caso de los países de América Latina y en la región, en promedio, se ha documentado que la desigualdad tuvo una tendencia creciente en las dos últimas décadas del siglo XX, que se revirtió a partir del año 2000 (Alvaredo y Gasparini 2015; De Ferranti et al. 2004; De la Torre et al., 2014; Gasparini y Lustig 2011; López-Calva y Lustig, 2010; De la Torre et al., 2017). Uno de los argumentos centrales de Gasparini y Lustig (2011) para explicar el aumento de la desigualdad en el primer periodo, es que las reformas que se llevaron acabo como respuesta a las crisis afectaron desproporcionadamente a las clases bajas y a la clase media, además de que en ese periodo los mecanismos de seguridad para los grupos más vulnerables eran casi inexistentes o estaban mal diseñados. Sin embargo, aun cuando pareciera que, a partir del 2000, la tendencia de la desigualdad comenzó a ser decreciente, los datos que reportan para los casos particulares de Argentina, Brasil y México, muestran un comportamiento contracíclico: en los periodos de crisis, la desigualdad se incrementa y en los periodos subsecuentes, vuelve a disminuir frente a la recuperación económica, lo que indica que en promedio hay una mejor recuperación de los grupos de ingresos bajos. Si bien esto tiene que ver con las políticas gubernamentales donde las transferencias juegan un papel importante, los cambios en la retribución del trabajo han sido también un factor determinante. Un argumento importante en los trabajos enfocados en América Latina es el De la Torre et al. (2017), quienes proponen que la disminución de la desigualdad -se debe en buena medida- a un aumento en el pago de trabajo no calificado y a una disminución de la retribución a los trabajadores con mayor educación, que ha causado una disminución en la inequidad del ingreso salarial. Sin embargo, durante las crisis, el desempleo afecta con más intensidad a los trabajadores con menores ingresos, por lo que se esperaría una caída de mayor proporción en los salarios de los trabajadores menos calificados y un consecuente incremento en la desigualdad, por ingreso salarial de los hogares. Esto es consistente con el aumento en la desigualdad del componente salarial del ingreso que encontramos y presentamos en este trabajo para el caso de México durante el periodo de la Gran Recesión. Este comportamiento refleja en buena medida la dinámica de lo que ocurre en el mercado de trabajo formal y que

2 México se encuentra entre el grupo de países que aumentan su desigualdad durante y después de una recesión cuando se utiliza un análisis de ciclos económicos tradicionales. Cuando se consideran ciclos de crecimiento, México se encuentra entre los países que tienen un aumento en la desigualdad después de tres años de la recesión, pero no antes. 
podría explicar la tendencia que se observa en los países de mayores ingresos (Camacho y Palmieri, 2019). Esta tendencia, no obstante, no se mantiene en nuestras medidas de ingreso total por trabajo, que no solo incluye el ingreso salarial, sino el ingreso por negocios y autoempleo, y que, en contraste, incluye las retribuciones que ofrece el sector informal.

En el caso particular de la Gran Recesión diferentes trabajos observan un incremento en la desigualdad tanto en el ingreso como en la riqueza en los Estados Unidos (Meyer y Sullivan, 2013; Wolf, 2013; Pfeffer et al., 2013; Emmons y Noeth, 2012; Piketty y Saez, 2013; U.S. Congress, 2010; Perri y Steinberg, 2012). Estos análisis reportan que la crisis impactó con más fuerza a los sectores con ingresos y riqueza más bajos. Wolf (2013), por ejemplo, señala que entre 2007 y 2010 el Top_10 aumentó en $0.2 \%$ su proporción de riqueza total, mientras que el Bot_40 tuvo una disminución de $1.1 \%$, lo que provocó un aumento de la desigualdad medida en el coeficiente de Gini de 0.834 a $0.870 .{ }^{3}$ En el caso del ingreso personal para el Top_10, el incremento fue de $0.4 \%$, y para el Bot_40 de -1.7\%. Pfeffer et al. (2013), sostienen que durante la Gran Recesión todos los grupos socioeconómicos sufrieron disminuciones en su ingreso y su riqueza, sin embargo, en términos proporcionales los grupos más afectados fueron los menos aventajados en términos de su pertenencia a minorías, niveles educativos, así como el nivel de ingreso del que disponían antes de la recesión. En el mismo sentido, Emmons y Noeth (2012) documentan una disminución del $26 \%$ de la riqueza de los hogares en Estados Unidos, durante mediados del 2007 y principios del 2009. En su análisis muestran, que: en términos porcentuales, los más afectados por la crisis fueron familias jóvenes con jefes menores de 40 años $(-43.8 \%$ de su ingreso total, contra menos del $-20 \%$ del resto), en desagregados por raza o etnicidad, la mayor pérdida fue para familias afro-americanas e hispanas ($37.2 \%$ de su ingreso total, contra $-11 \%$ del resto) y en términos educativos, los más afectados fueron los hogares con grado no universitario $(-22.3 \%$ de su ingreso total, contra $-15.5 \%$ de quienes sí cuentan con grado universitario).

Sin embargo, la Gran Recesión no impactó los índices de desigualdad en la misma dirección en todos los países. En algunos casos, como el de Suecia, Alemania y Reino Unido, la distribución del ingreso prácticamente no cambió o, incluso en casos como el de Irlanda, se redujo en los años subsecuentes al inicio de la crisis (Grabka, 2015; Jenkins, Brandolini, Micklewright, Nolan, y Basso, 2013).

Aunque nuestro trabajo no se enfoca en la distribución de la riqueza debido a la escases de datos, es importante mencionar que, en general, se ha observado aumento en la desigualdad para la mayoría de los países europeos durante la

3 Usamos el termino Top_10 para referirnos al 10\% de la población con mayores ingresos, mientras que el Bot_40 se refiere al $40 \%$ con menores ingresos. 
Gran Recesión, aunque hay también algunas excepciones como Bélgica y Eslovaquia (Bogliacino y Maestri, 2016).

En el caso de Alemania, Grabka (2015) señala que no existieron cambios distribucionales significativos durante la Gran Recesión en este país. No obstante, dada la dificultad de identificación como en la mayoría de los casos, el análisis de Grabka (2015) no aísla el efecto de la crisis de las respuestas de política, y considera que el comportamiento que observa se debe a la pronta reacción del gobierno alemán con la creación de estímulos económicos, y a factores como la pronta recuperación del PIB por la alta demanda global de las exportaciones alemanas, y a la flexibilidad en el mercado laboral, que permitió conservar trabajadores calificados bajo la expectativa de la recuperación y el aumento en la demanda de bienes manufacturados.

En otro trabajo, que analiza los cambios de la distribución del ingreso para 21 países miembros de la OCED a partir de la Gran Recesión, Jenkins, et al. (2013), identifican efectos en las dos direcciones. ${ }^{4}$ Encuentran que en 10 países existió una ligera caída de la desigualdad durante ese periodo, siendo Irlanda el caso más importante, para el que calculan una disminución del coeficiente de Gini de $2 \%$ mientras que en países como Francia y España el coeficiente tuvo un incremento de $3.5 \%$ y $1 \%$, respectivamente. En sus conclusiones, plantean: "el panorama general es que no existe una tendencia universal, creciente o decreciente, sobre el comportamiento de la desigualdad, al menos durante los dos años posteriores al inicio de la GR" (Jenkins et al., 2013, pp. $2-10)$.

Para el caso latinoamericano, la mayoría de los estudios no se enfocan específicamente en la Gran Recesión, pero aquellos que alcanzan a cubrir ese periodo sostienen que la tendencia decreciente de la desigualdad se mantuvo, a pesar de los efectos de la Gran Recesión (Alvaredo y Gasparini 2015; De la Torre et al., 2014; De la Torre et al., 2017; Domínguez y García, 2016).

Comparativamente, ante el caso norteamericano, la literatura que discute el impacto redistributivo que la Gran Recesión tuvo en el caso mexicano, es escaza. Los trabajos relacionados con los efectos que tuvo la Gran Recesión en México se han centrado sobre todo en los impactos en el mercado laboral, la pobreza y la producción a nivel estatal o regional, pero no en los cambios distribucionales. Algunos de los estudios que dan seguimiento y discuten la evolución de la desigualdad en México, abarcan el periodo de la contracción correspondiente a los efectos de la Gran Recesión; todos ellos, y aunque no se detienen en analizar este periodo en detalle, reportan una disminución de los

4 En su estudio considera Australia, Austria, Bélgica, Canadá, Dinamarca, Alemania, Grecia, Finlandia, Francia, Irlanda, Italia, Japón, Países Bajos, Nueva Zelanda, Noruega, Portugal, España, Suecia, Suiza, Reino Unido, y Estados Unidos. 
indicadores de inequidad entre 2008 y 2010 (Cortés, 2013; Cortés y Vargas, 2017; Iniguez-Montiel y Kurosaki, 2018). Esto, aun cuando en general no hay un patrón definido sobre los efectos de la crisis sobre la desigualdad, llama la atención para el caso mexicano.

Aún en el caso de estudios que, al ajustar los datos de la ENIGH con los de Cuentas Nacionales para corregir problemas de subregistro, reportan una tendencia claramente creciente de la desigualdad después del 2000, se observa también una caída de la desigualdad del ingreso entre 2008 y 2010 (Del Castillo, 2015). Cortés y Vargas (2017), en una importante discusión sobre la medición de la desigualdad en México a lo largo de 50 años, reportan una disminución del índice de Gini para el ingreso corriente de los hogares de 2008 a 2010 , de 0.471 a 0.446 , y un ligero aumento en 2012 a 0.452 . De igual forma, para el periodo 2008-2010, reportan una disminución de la razón del ingreso medio per cápita del decil X respecto al decil I de 26 a 23 unidades. Aun cuando estas mediciones se refieren al ingreso corriente, que considera el valor de la renta por la propiedad de la vivienda y el ingreso en especie, concuerdan con la disminución del ingreso monetario de los hogares que nosotros reportamos. Esto implica que, si bien componentes del ingreso corriente como el valor de la renta y el ingreso en especie disminuyen el índice de Gini, no son los que definen la tendencia decreciente de la desigualdad del ingreso de los hogares, durante ese periodo. Esto contrasta con el aumento en la desigualdad que reportamos en este trabajo, para los ingresos por salario, y su disminución para nuestra dimensión de ingreso total por trabajo, cuya magnitud es muy pequeña.

Los choques económicos que afectan las posibilidades de los hogares para generar sus ingresos en distintos mercados dependen de factores estructurales e institucionales no solo en el nivel nacional, sino también en el nivel regional. Un importante número de estudios han dado cuenta de las diferencias regionales de la dinámica económica en el país, y aunque son menos los que se han enfocado a estudiar las diferencias en el proceso distributivo, sabemos de las importantes disparidades de desarrollo en México, y los impactos sobre los niveles de pobreza y desigualdad (Esquivel y Messmacher, 2002; González, 2007; Gutiérrez, 2008; Esquivel y Cruces, 2011; Chiquiar, 2008; Lustig, 2010; Ros, 2015). Así, por ejemplo, tanto la desigualdad del ingreso en general (Gutiérrez, 2008), como la desigualdad salarial (Chiquiar, 2008) han tendido a disminuir en las regiones más expuestas a la apertura comercial. Sin embargo, frente a un choque exógeno como la Gran Recesión, la contracción de la demanda de trabajo en la industria manufacturera, ligada fuertemente con el comercio exterior, puede tener un efecto mayor en la desigualdad en aquellas regiones donde se concentra más este tipo de industria, y principalmente en la manufactura que demanda menor calificación en el trabajo. 
Aunque sin indagar en la distribución del ingreso, Espinal (2010) efectivamente señala cómo los estados en los que el PIB se compone principalmente de actividades manufactureras sufrieron un mayor impacto frente a la crisis, que aquellos donde la producción manufacturera tiene un menor peso. De esta forma, esperaríamos no solo impactos diferentes en magnitud para las distintas regiones, sino también dinámicas diferenciadas sobre los ingresos por salarios y los ingresos por negocios y autoempleo. Aun cuando es importante tener una mejor idea de las diferencias regionales en cuanto a las dinámicas de la distribución del ingreso que pueden ser analizadas con un poco más de claridad, durante eventos como el de la Gran Recesión, no existe literatura que indague sobre la dinámica de la desigualdad para distintos componentes del ingreso de los hogares en nivel regional. Aunque el objetivo de este trabajo no es hacer un análisis exhaustivo del tema, presentamos una aproximación muy general que evidencia diferencias importantes para las distintas regiones del país.

Así, consideramos que además de proveer un análisis sobre el impacto que la contracción económica de 2008-2010 -causada por la Gran Recesión- tuvo en la distribución del ingreso de los hogares y de algunos de sus componentes, los resultados que presentamos en este trabajo, aportan elementos a la literatura que busca comprender la forma en como los choques macroeconómicos impactan los ingresos de los hogares en diferentes estratos y para distintos grupos socioeconómicos, ya sea tanto en nivel nacional como regional.

\section{Descripción y construcción de variables}

La información utilizada en este análisis proviene de las Encuestas Nacionales de Ingreso Gasto de los Hogares (ENIGH) de los años 2006 a 2018. Los valores monetarios se transformaron en valores reales a precios de 2018 utilizando el INPC estatal, para indexar los ingresos de cada hogar, de acuerdo con el nivel de precios calculado para la entidad federativa a la que pertenece.

Para cada hogar consideramos tres dimensiones de ingreso que corresponden a diferentes fuentes. La más simple es la que corresponde al pago por trabajo subordinado o asalariado, y que llamamos ingreso por trabajo asalariado (IS). Por sus características como pago por trabajo subordinado, este ingreso tiene una mayor relación con el mercado formal y puede reflejar con mayor cercanía la dinámica de ese sector en comparación con otros ingresos, como los provenientes del autoempleo y los negocios propios. El ingreso por salarios, si bien no tiene necesariamente una correlación perfecta con la productividad del trabajo debido a las distorsiones de los mercados, sí refleja la valoración del trabajo en el mercado y permite observar su dinámica en distintos estratos socioeconómicos y los efectos que tienen diferentes factores en esa valoración $y$, por ende, en su distribución y en la desigualdad. 
La segunda dimensión es la que llamamos ingresos totales por trabajo (ITT), que además de incluir el ingreso que reciben los hogares por trabajo subordinado también considera los ingresos por trabajo independiente, ya sea como autoempleo o por negocios propios (N). El ingreso total por trabajo se compone, entonces, por la suma de los conceptos de ingresos por trabajo (IS) $\mathrm{y}$ de negocios $(\mathrm{N})$ :

$\mathrm{ITT}=\mathrm{IS}+\mathrm{N}$

Estos conceptos se componen de la siguiente manera:

- Ingresos por trabajo (IS): Sueldos, salarios o jornal, destajo, comisiones y propinas, horas extras, incentivos, gratificaciones o premios, bonos, percepciones adicionales o sobresueldos, primas vacacionales y otras prestaciones en efectivo, reparto de utilidades y aguinaldo. Tanto por trabajo principal como por trabajo secundario.

- Ingresos por negocios (N): Se incluyen negocios de actividades industriales o comerciales, prestación de servicio, actividades agrícolas, cría y explotación de animales, recolección, reforestación y tala de árboles, pesca, caza y captura de animales. Tanto por trabajo principal como por trabajo secundario.

El ITT es un indicador de la remuneración al trabajo que en el caso de México incluye un componente muy importante de los ingresos por autoempleo y por servicios y comercio vinculados al sector informal, particularmente en los deciles de ingreso bajo. No obstante, hay que ser muy cautelosos con la interpretación de estos indicadores, ya que la información de la ENIGH no nos permite separar los ingresos provenientes de la informalidad de aquellos ligados a los mercados formales.

El ingreso total con el que disponen los hogares para tomar sus decisiones de consumo y ahorro se compone además de los pagos al trabajo y remuneraciones por negocios de las transferencias que reciben como remesas o subsidios de programas sociales, así como rentas e ingresos provenientes de activos en posesión de los hogares. Este ingreso es lo que llamamos Ingreso Monetario (IM) y se compone con los ingresos por concepto de trabajo (trabajo subordinado), de negocios (trabajo independiente), de transferencias de todos los tipos (TR), rentas (R) y otros ingresos (OI):

$\mathrm{IM}=\mathrm{IS}+\mathrm{N}+\mathrm{TR}+\mathrm{R}+\mathrm{OI}$

donde: 
- Rentas (R): Se incluyen los ingresos por alquiler de tierras, terrenos, casas, edificios, locales y otros inmuebles, tanto dentro del país como fuera de él. Además de los intereses provenientes de inversiones a lago plazo, cuentas de ahorro y prestamos a terceros, así como por rendimientos de bonos o cedulas, alquiler de marcas, patentes o derechos de autor. Se incluyen también las ganancias y utilidades producto de la participación en cooperativas, sociedades y empresas que funcionan como sociedades.

- Transferencias (TR): Las transferencias incluyen: indemnizaciones de seguros por accidentes de trabajo, por despido y retiro voluntario, becas de instituciones privadas y donativos de organizaciones no gubernamentales, donativos de otros hogares, todas estas etiquetadas como otras transferencias (OTR), ingresos de otros países (REM), beneficios de programas sociales y becas de gobierno (TRG).

- Otros ingresos (OI): Cualquier otro ingreso extra que tenga el hogar.

En este trabajo, se busca describir los efectos de un choque sobre la capacidad de los hogares, para obtener sus ingresos a partir de distintas fuentes, y dado que no nos enfocamos propiamente en analizar los efectos sobre el bienestar y su distribución, a diferencia de otros análisis que observan el ingreso corriente de los hogares, no incluimos en nuestras categorías la estimación de la renta por la propiedad de la vivienda que habita el hogar. Al enfocarnos en la capacidad que tiene cada hogar para proveerse de ingresos como una unidad, para el análisis de las diferentes dimensiones, agregamos el ingreso de todos los integrantes del hogar y calculamos el promedio mensual de cada una de sus dimensiones. En esta agregación, descontamos los ingresos estacionales y extraordinarios como bonos y aguinaldos, de tal forma que las variables de ingreso que construimos sean comparables para todos los hogares, hayan o no recibidos pagos por prestaciones como las mencionadas durante los periodos del levantamiento de la encuesta.

La ENIGH ha tenido diversos cambios metodológicos desde su creación, sin embargo, el más importante fue en 2016, cuando se amplió la muestra para obtener resultados con representatividad a nivel estatal. Si bien, las ediciones anteriores a la de 2016 no son representativas a nivel estatal, constituyen la única fuente de información sobre el ingreso de los hogares a nivel estatal, por lo que es la mejor aproximación con la que contamos.

Las principales estadísticas de nuestras diferentes dimensiones de ingreso y su composición se muestran en las tablas 1 y 2. 
Tabla 1

Estadísticas principales de las diferentes dimensiones del ingreso mensual (Pesos constantes 2018)

\begin{tabular}{llllllllllll}
\hline \multirow{2}{*}{ Año } & \multirow{3}{*}{ Obs. } & Media & \multicolumn{4}{c}{ Mediana } & \multicolumn{4}{c}{$\begin{array}{c}\text { Coeficiente } \\
\text { Variación }\end{array}$} & de \\
& & IM & ITT & IS & IM & ITT & IS & IM & ITT & IS \\
\hline $\mathbf{2 0 0 6}$ & 20,853 & 14,306 & 10,852 & 9,209 & 9,550 & 7,415 & 5,971 & 1.28 & 1.26 & 1.43 \\
$\mathbf{2 0 0 8}$ & 29,468 & 14,339 & 11,174 & 8,845 & 9,150 & 7,286 & 5,817 & 1.76 & 1.98 & 1.39 \\
$\mathbf{2 0 1 0}$ & 27,593 & 12,456 & 9,640 & 8,214 & 8,378 & 6,434 & 5,259 & 1.22 & 1.33 & 1.49 \\
$\mathbf{2 0 1 2}$ & 8,985 & 12,522 & 9,372 & 7,766 & 8,170 & 6,128 & 4,845 & 1.34 & 1.43 & 1.51 \\
$\mathbf{2 0 1 4}$ & 19,458 & 12,232 & 9,810 & 8,337 & 8,052 & 6,391 & 5,231 & 1.77 & 2.09 & 1.67 \\
$\mathbf{2 0 1 6}$ & 70,267 & 13,848 & 9,825 & 8,572 & 9,128 & 6,748 & 5,707 & 6.08 & 1.43 & 1.37 \\
$\mathbf{2 0 1 8}$ & 74,597 & 13,272 & 9,909 & 8,599 & 9,291 & 7,082 & 5,902 & 1.48 & 1.29 & 1.38 \\
\hline
\end{tabular}

Fuente: Elaboración propia con información de la ENIGH, diferentes años.

Tabla 2

Valor promedio de los componentes de las diferentes dimensiones del ingreso mensual (Pesos constantes 2018)

\begin{tabular}{llllllll}
\hline Año & $\begin{array}{l}\text { Trabajo } \\
\text { (IS) }\end{array}$ & $\begin{array}{l}\text { Negocio } \\
\text { (N) }\end{array}$ & $\begin{array}{l}\text { Rentas } \\
\text { (R) }\end{array}$ & $\begin{array}{l}\text { Transfe } \\
\text { rencias } \\
\text { Gobiern } \\
\text { o } \\
\text { (TRG) }\end{array}$ & $\begin{array}{l}\text { Remesas } \\
\text { (REM) }\end{array}$ & $\begin{array}{l}\text { Otras } \\
\text { Transfe } \\
\text { rencias } \\
\text { (OTR) }\end{array}$ & $\begin{array}{l}\text { Otros } \\
\text { (OI) }\end{array}$ \\
\hline $\mathbf{2 0 0 6}$ & 9,209 & 1,642 & 1,633 & 220 & 329 & 1,263 & 10 \\
$\mathbf{2 0 0 8}$ & 8,845 & 2,329 & 865 & 293 & 188 & 1,340 & 12 \\
$\mathbf{2 0 1 0}$ & 8,214 & 1,426 & 561 & 307 & 151 & 1,355 & 15 \\
$\mathbf{2 0 1 2}$ & 7,766 & 1,607 & 661 & 305 & 121 & 1,601 & 19 \\
$\mathbf{2 0 1 4}$ & 8,337 & 1,473 & 203 & 350 & 95 & 1,357 & 16 \\
$\mathbf{2 0 1 6}$ & 8,572 & 1,253 & 1,513 & 345 & 128 & 1,598 & 15 \\
$\mathbf{2 0 1 8}$ & 8,599 & 1,310 & 973 & 274 & 131 & 1,559 & 15 \\
\hline
\end{tabular}

Fuente: Elaboración propia con información de la ENIGH, diferentes años.

\section{La desigualdad de las dimensiones del ingreso}

De acuerdo con los datos de la tabla 1, a partir de 2008, los promedios para las tres dimensiones de ingreso tuvieron una caída importante y continuaron decreciendo durante varios años, para comenzar a recuperarse en 2014.

De 2008 a 2010, las medianas del ingreso total por trabajo (ITT) y del ingreso monetario (IM) cayeron en menor proporción que sus respectivas medias, y sus coeficientes de variación también disminuyeron, lo cual indica que, en promedio, estas dimensiones de ingreso cayeron en una proporción menor en los deciles bajos que en los altos. No obstante, en el caso del ingreso por 
salarios (IS), ocurrió lo contrario y los hogares de los cinco primeros deciles fueron los que tuvieron una mayor pérdida.

Los distintos comportamientos del ingreso por salarios y del ingreso total por trabajo, están acompañados por una caída importante de los ingresos por negocios (tabla 2), lo que indica que está última caída afectó sobre todo a los hogares en los deciles altos.

De los componentes que diferencian el ingreso total por trabajo y el ingreso monetario, entre 2008 y 2010, solo cayeron los promedios de las remesas y de las rentas. De acuerdo con la tabla 2, mientras que las remesas cayeron en $19.68 \%$, las rentas tuvieron una disminución de $35.14 \%$, lo cual, junto con la contracción de los ingresos por negocios, explica que el ingreso monetario haya tenido en promedio una mayor contracción para los hogares en los deciles por arriba de la media.

Los mayores efectos que tuvo la contracción en el ingreso monetario y el ingreso total del trabajo para los hogares en los deciles altos se pueden observar en la tabla 3.

En 2008, el ingreso monetario promedio de los hogares que se encuentran en el Bot_40 de la distribución, fue de $\$ 4,171$; mientras que, en 2010 , fue de $\$ 3,868$, lo que implica una diminución de $7.2 \%$. En el caso del promedio del ingreso monetario para los hogares del Top_10, el cambio entre el año 2008 y el 2010 fue de $-18.2 \%$, es decir, la contracción del ingreso monetario promedio del Top_10 fue casi 2.5 veces que la que tuvo el Bot_40 (tabla 3 ).

Tabla 3

Evolución del ingreso mensual en sus diferentes dimensiones por grupo poblacional

\begin{tabular}{|c|c|c|c|c|c|c|c|c|c|c|c|c|}
\hline \multirow{3}{*}{ Año } & \multicolumn{6}{|l|}{ Top_10 } & \multicolumn{6}{|c|}{ Bot_40 } \\
\hline & \multicolumn{2}{|c|}{$\begin{array}{l}\text { Ingreso } \\
\text { Monetario }\end{array}$} & \multirow{2}{*}{$\begin{array}{l}\begin{array}{l}\text { Ingreso } \\
\text { Trabajo }\end{array} \\
\text { Pesos }\end{array}$} & \multirow{2}{*}{$\begin{array}{c}\text { Total } \\
\Delta \%\end{array}$} & \multicolumn{2}{|c|}{ Ingreso Salario } & \multicolumn{2}{|c|}{$\begin{array}{l}\text { Ingreso } \\
\text { Monetario }\end{array}$} & \multirow{2}{*}{$\begin{array}{l}\begin{array}{l}\text { Ingreso } \\
\text { Trabajo }\end{array} \\
\text { Pesos }\end{array}$} & \multirow{2}{*}{$\begin{array}{r}\text { Total } \\
\Delta \%\end{array}$} & \multicolumn{2}{|c|}{ Ingreso Salario } \\
\hline & Pesos & $\Delta \%$ & & & Pesos & $\Delta \%$ & Pesos & $\Delta \%$ & & & Pesos & $\Delta \%$ \\
\hline 2006 & 52,811 & & 42,444 & & 42,609 & 5.1 & 4,549 & & 3,643 & & 3,858 & 6.17 \\
\hline 2008 & 54,803 & 3.77 & 46,231 & 8.9 & 39,627 & -7.0 & 4,171 & -8.3 & 3,384 & -7.12 & 3,552 & -7.9 \\
\hline 2010 & 44,777 & -18.2 & 39,419 & -14.7 & 39,080 & -1.3 & 3,868 & -7.2 & 3,105 & -8.24 & 3,357 & -5.4 \\
\hline 2012 & 47,318 & 5.68 & 39,695 & 0.7 & 37,757 & -3.3 & 3,656 & -5.4 & 2,684 & -13.5 & 3,038 & -9.5 \\
\hline 2014 & 46,140 & -2.49 & 42,355 & 6.7 & 40,499 & 7.26 & 3,754 & 2.6 & 2,882 & 7.37 & 3,200 & 5.31 \\
\hline 2016 & 52,840 & 14.52 & 38,097 & -10.0 & 37,568 & -7.2 & 4,305 & 14.67 & 3,260 & 13.09 & 3,493 & 9.17 \\
\hline 2018 & 46,519 & -11.9 & 37,766 & -0.87 & 37,256 & -0.8 & 4,396 & 2.11 & 3,441 & 5.57 & 3,708 & 6.15 \\
\hline
\end{tabular}

Fuente: Elaboración propia con información de la ENIGH, varios años. 
En el caso del ingreso total del trabajo, que incluye los salarios y los ingresos por negocios, la disminución para el Top_10 fue de 14.7\%, mientras que para el Bot_40, fue de $8.24 \%$. La disminución que en este caso sufrió el Bot_40, es mayor que en el caso del ingreso total por trabajo, lo cual confirma que la pérdida de ingreso por negocios y por rentas se concentró en los deciles altos, además, las transferencias gubernamentales, que se concentran en los hogares de menor ingreso, también ayudaron a mitigar la caída. Por esta misma razón, la pérdida sufrida por el Top_10 es mayor en el ingreso monetario que la que podemos observar en el ingreso total por trabajo.

La única dimensión donde el impacto fue mayor en el Bot_40 que en el Top_10, fue en el ingreso por salarios. En el caso del ingreso por salarios, el Bot_40 experimentó una reducción del 5.4\%; mientras que en el Top_10, la disminución fue únicamente de un $1.3 \%$.

El impacto negativo de la Gran Recesión sobre el ingreso monetario en el Top_10 aún es visible, pues estos hogares no han logrado recuperar el nivel de ingresos que tenían en 2008, lo mismo sucede con el ingreso total del trabajo. Esta falta de recuperación en las dos dimensiones está asociada con la tendencia decreciente que se observa en el ingreso por negocios (tabla 2) y en el caso del ingreso monetario, con la dinámica de las rentas (R) (ver tabla 2). Mientras tanto, en el caso de los ingresos salariales se observó una recuperación en 2014, donde los ingresos de esta dimensión fueron superiores a los que se tenían en 2008, sin embargo, ese impacto positivo perdió fuerza, pues en los 4 años siguientes experimentaron una caída de $7.2 \%$ en los dos primeros años, y de $0.8 \%$ los siguientes dos años para, finalmente, terminar por debajo de su nivel de 2008.

La historia sobre la recuperación de los hogares que se ubican en el Bot_40 es distinta, ya que desde 2016 el ingreso monetario promedio ha sido mayor que lo que tenían en el 2008, mientras que en el caso del ingreso total del trabajo y del ingreso salarial, la recuperación recién se observa en 2018. Así, hasta el año 2018, los hogares del decil 10 no habían logrado recuperarse del impacto negativo que tuvo la crisis en sus ingresos, mientras que en el caso del Bot_40, logró rebasar los niveles de ingreso para las tres dimensiones que tenía en 2008.

Una de las posibles causas de la mejor recuperación del ingreso monetario para el Bot_40 podría estar relacionada con las transferencias, principalmente las que se hacen entre hogares. De acuerdo con los datos de la ENIGH, las transferencias gubernamentales tuvieron un incremento importante; durante 2008, el promedio de ingresos mensuales por transferencias provenientes del gobierno que recibieron los hogares en México fue de 293; mientras que para 2010 tuvieron un incremento de casi 5\%, con una ligera disminución en 2012, para recuperase en el año 2014 con un incremento de $15 \%$. Por el contrario, en el caso de las remesas, se observa una disminución sostenida desde 2008, con 
ligeras recuperaciones; pero, en general, las remesas promedio que reciben los hogares, no ha logrado situarse en los niveles anteriores a la Gran Recesión. Otro de los componentes de las transferencias es "otras transferencias", las cuales incluyen principalmente jubilaciones y transferencias de otros hogares. Como puede observarse en la tabla 2, la magnitud de estas transferencias es significativamente más alta que la de las remesas y las transferencias gubernamentales en todos los años. Si bien este tipo de transferencias sufrieron variaciones importantes en el periodo de la crisis, han logrado recuperarse a niveles incluso superiores a los que tenían antes de la Gran Recesión. Así, los incrementos de las transferencias gubernamentales, y de "otras transferencias", están estrechamente relacionadas con el aumento que se observa en el ingreso monetario y su recuperación para el año 2016.

En resumen, la contracción económica tuvo un mayor impacto negativo en el ingreso salarial en los hogares con menores ingresos; sin embargo, cuando se consideran los ingresos totales por trabajo que además de incluir el pago por trabajo subordinado, también incluye los negocios propios y el autoempleo, el mayor impacto se registra en los hogares de ingresos altos. Finalmente, al incluir ingresos provenientes de fuentes distintas al trabajo, el efecto que se observa para el ingreso total por trabajo se refuerza. Estos impactos permanecen durante los años posteriores a la contracción, y se observa una mejor recuperación para los hogares en los deciles bajos que para los del decil más alto, incluyendo los ingresos por salario.

Esta dinámica se refleja en el comportamiento de diferentes indicadores de desigualdad. La gráfica 1 presenta la estimación de diferentes medidas de desigualdad para cada una de las dimensiones del ingreso. En la gráfica correspondiente a los índices de Gini, se puede observar durante el periodo 2008-2010 un aumento para el caso del ingreso por salarios (IS), que contrasta con una ligera disminución de la desigualdad del ingreso total del trabajo (ITT), y una caída aún más pronunciada en la desigualdad del ingreso monetario (IM). Estos distintos cambios en la desigualdad de las tres dimensiones reflejan las dinámicas que ya hemos analizado y descrito, en los párrafos anteriores.

Dadas las limitaciones que tiene el índice de Gini ante cambios en los extremos de la distribución, estimamos también el índice de Theil, para robustecer nuestro análisis. En el caso del ingreso por salarios, entre 2008 y 2010, el índice de Gini creció en $2.1 \%$, mientras que el Índice de Theil también tuvo un aumento de $6.0 \%$. En cuanto a la proporción del total de los ingresos salariales concentrados en el Bot_40, esta cayó 7.8\% mientras que la del Top_10 aumentó $4.5 \%$, y podemos ver en la tabla 4 que el aumento de la concentración fue mayor en los hogares de los últimos cinco percentiles y más, aún, en el 
último percentil (ver tabla 4). Todas estas medidas son consistentes y muestran un aumento de la desigualdad en el ingreso por salarios.

\section{Grafica 1}

Evolución del Top_10 y Bot_40 sobre las diferentes dimensiones

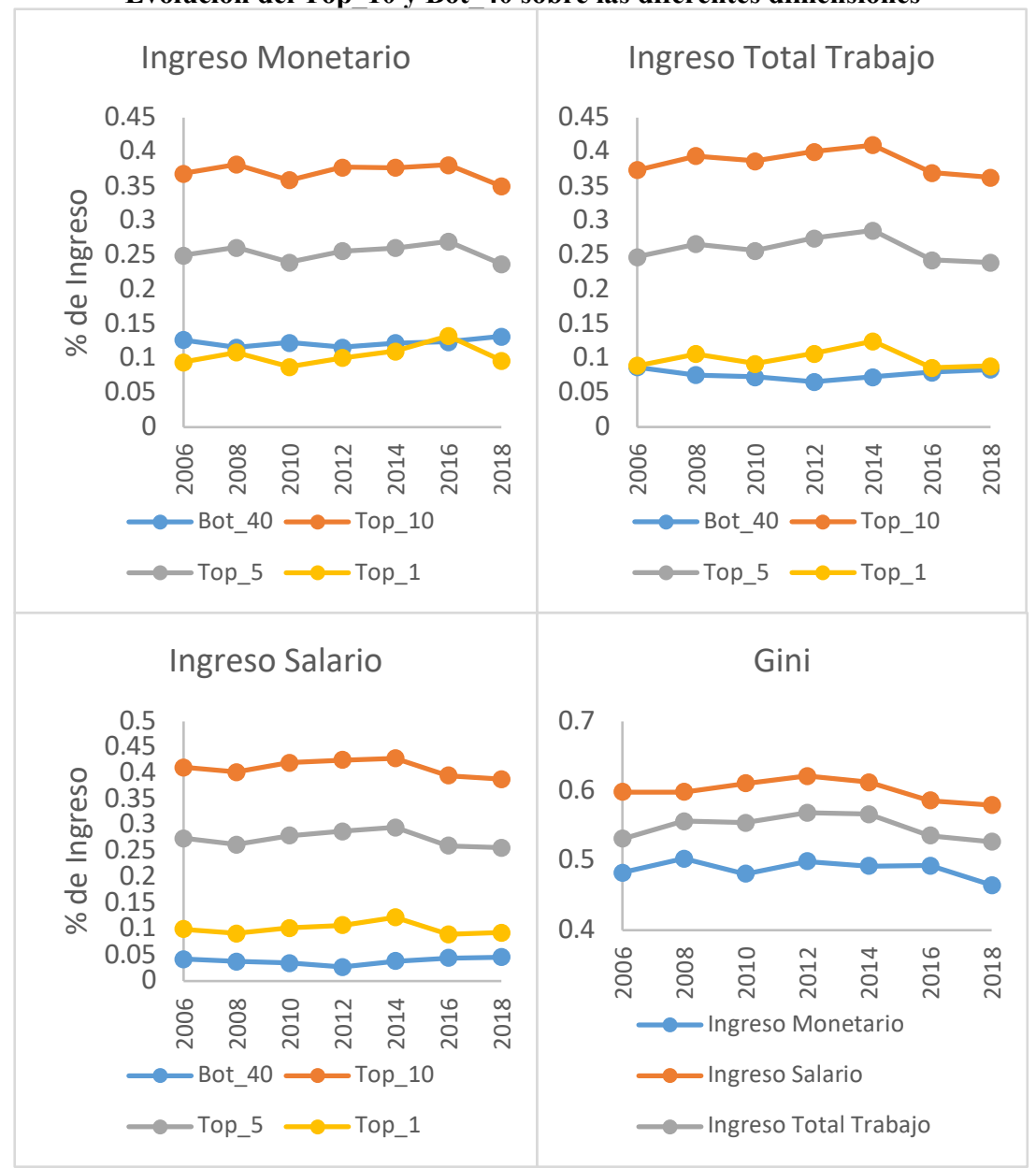

Fuente: Elaboración propia con información de la ENIGH, diferentes años.

En el mismo periodo, el coeficiente de Gini correspondiente al ingreso total del trabajo tuvo una disminución muy pequeña de $0.4 \%$, mientras que el índice de Theil cayó $12 \%$, y los 4 grupos poblacionales experimentaron también una caída de sus participaciones en el ingreso total. La mayor afectación fue para el Top_1, con -13\% (ver tabla 4), lo cual refleja cómo la caída en los ingresos por negocios fue más importante en los hogares de ingresos altos. Este efecto 
no sólo atenuó el aumento en la desigualdad de los salarios, sino que logró revertirlo de tal forma que la inequidad del ingreso total por trabajo disminuyera levemente.

En cuanto al ingreso monetario, en 2008 el coeficiente de Gini fue de 0.5029 y para 2010 se ubicó en 0.4811 ; una reducción en términos porcentuales de 4.3\%. De igual manera, el índice de Theil disminuyó en $12.0 \%$. En contraste con el ingreso total del trabajo, en este caso, no solo las pérdidas en los Top_10, 5 y 1 fueron mayores, sino que para el Bot 40, se registró un aumento. Estas disminuciones del coeficiente de Gini y del índice de Theil, correspondientes al ingreso monetario durante 2008-2010, se debieron a una mayor caída de la proporción de ingresos de los deciles superiores, en relación con el impacto experimentado por los hogares en los deciles inferiores. Este fenómeno podría considerarse una tendencia a la igualdad por empobrecimiento, como sostiene Cortés (2012). En este periodo, la proporción de los ingresos monetarios totales que tenían los hogares del Bot_40 pasó de $11.6 \%$ a $12.3 \%$, reflejando un incremento de $6 \%$. En el otro extremo, la proporción del ingreso monetario de los hogares en el Top_1, Top_5 y Top_10 disminuyó en 17\%, 7\% y 5\%, respectivamente (ver tabla 4$)$.

Tabla 4

Cambio porcentual en la proporción de ingresos, Coeficiente de Gini y el Índice de Theil en cada dimensión durante 2008-2010

\begin{tabular}{|c|c|c|c|c|c|c|}
\hline Dimensión & Gini & Theil & Bot_40 & Top_10 & Top_5 & Top_1 \\
\hline $\begin{array}{l}\text { Ingreso } \\
\text { Monetario }\end{array}$ & $-4.3 \%$ & $-15.3 \%$ & $6.1 \%$ & $-6.0 \%$ & $-8.3 \%$ & $-19.3 \%$ \\
\hline $\begin{array}{l}\text { Ingreso } \\
\text { Total } \\
\text { Trabajo }\end{array}$ & $-0.4 \%$ & $-12.0 \%$ & $-3.6 \%$ & $-1.8 \%$ & $-3.7 \%$ & $-13.3 \%$ \\
\hline $\begin{array}{l}\text { Ingreso } \\
\text { Salario }\end{array}$ & $2.1 \%$ & $6.0 \%$ & $-7.8 \%$ & $4.5 \%$ & $6.6 \%$ & $11.3 \%$ \\
\hline
\end{tabular}

Fuente: Elaboración propia con información de la ENIGH, diferentes años.

Es importante destacar que la marcada caída de los indicadores de desigualdad para el ingreso monetario en el periodo 2008-2010, se debe principalmente a las transferencias entre los hogares y a las pensiones, es decir, no se debe a la retribución del trabajo contemporáneo de los hogares.

De manera similar con lo ocurrido entre 2008 y 2010, para el ingreso monetario, a partir del 2012, el coeficiente de Gini de las tres dimensiones de ingreso ha disminuido. Esta caída también está asociada con la baja en el porcentaje de ingresos del Top_10, Top_5 y en algunos casos del Top_1, mientras que el ingreso del Bot_40, se mantiene con ligeras variaciones poco perceptibles, como puede verse en la gráfica 1 . 
La tabla 5 muestra la evolución de tres índices de Palma (Palma, V2 y V3), que son las razones del porcentaje de ingresos del Top_10, Top_5 o Top_1, respecto al Bot_40, respectivamente. Estos indicadores nos permiten ver con más claridad los cambios que ocurrieron en las colas de la distribución, particularmente en la cola alta (Palma, 2011; Krozer, 2015). En el caso del ingreso salarial (IS), los tres indicadores de Palma aumentan, lo cual implica que hubo una mayor concentración en el decil 10 y que se distribuyó prácticamente en todos los percentiles de ese grupo. En el caso de la caída del ingreso por negocios, que afecta el ingreso total por trabajo (ITT), la historia es distinta. El índice de Palma aumentó de 2008 a 2010, mientras que el Palma V2 no cambió y el Palma V3 disminuyó, lo que indica que si bien aumentó la concentración del ingreso en el Top_10, el incremento relativo de la participación en el ingreso total se dio principalmente en los hogares del percentil 95 en adelante.

Si bien, en este caso, el índice de Palma para el ingreso total por trabajo (ITT) aumenta en esos años, el aumento es pequeño en relación con el aumento para el ingreso salarial (IS). Esto indica que la caída en los ingresos por negocios es proporcionalmente más importante para el Top_10, y aún cuando la diferencia en la caída del ingreso por negocios, entre los dos grupos, no logra hacer que el índice disminuya, sí mitiga por mucho el aumento en la desigualdad por salarios.

Finalmente, los tres indicadores para el caso del ingreso monetario (IM) disminuyen en el periodo. Esta disminución refleja el papel que las transferencias y la caída de las rentas tuvieron en el aumento de la participación del Bot_40, en el ingreso monetario total (IM) y en su disminución para el Top_10.

Tabla 5

Evolución de los índices de palma en las diferentes dimensiones

\begin{tabular}{llllllllll}
\hline \multirow{3}{*}{ Año } & \multicolumn{3}{l}{ Palma } & \multicolumn{4}{c}{ Palma V2 } & \multicolumn{4}{c}{ Palma V3 } \\
& ITT & IM & IS & ITT & IM & IS & ITT & IM & IS \\
\hline $\mathbf{2 0 0 6}$ & 4.31 & 2.91 & 9.70 & 2.85 & 1.97 & 6.48 & 1.03 & 0.75 & 2.36 \\
$\mathbf{2 0 0 8}$ & 5.22 & 3.29 & 10.73 & 3.53 & 2.25 & 7.01 & 1.41 & 0.94 & 2.45 \\
$\mathbf{2 0 1 0}$ & 5.32 & 2.92 & 12.15 & 3.53 & 1.95 & 8.10 & 1.27 & 0.71 & 2.95 \\
$\mathbf{2 0 1 2}$ & 6.09 & 3.25 & 15.64 & 4.17 & 2.20 & 10.59 & 1.62 & 0.87 & 3.96 \\
$\mathbf{2 0 1 4}$ & 5.61 & 3.08 & 11.27 & 3.91 & 2.13 & 7.78 & 1.71 & 0.90 & 3.22 \\
$\mathbf{2 0 1 6}$ & 4.63 & 3.08 & 8.89 & 3.05 & 2.18 & 5.85 & 1.08 & 1.07 & 2.03 \\
$\mathbf{2 0 1 8}$ & 4.35 & 2.65 & 8.48 & 2.87 & 1.80 & 5.61 & 1.06 & 0.73 & 2.04 \\
\hline
\end{tabular}

Fuente: Elaboración propia con información de la ENIGH, diferentes años. 


\section{Grafica 2}

Cambios en el ingreso promedio para diferentes puntos en el tiempo y diferentes grupos poblacionales
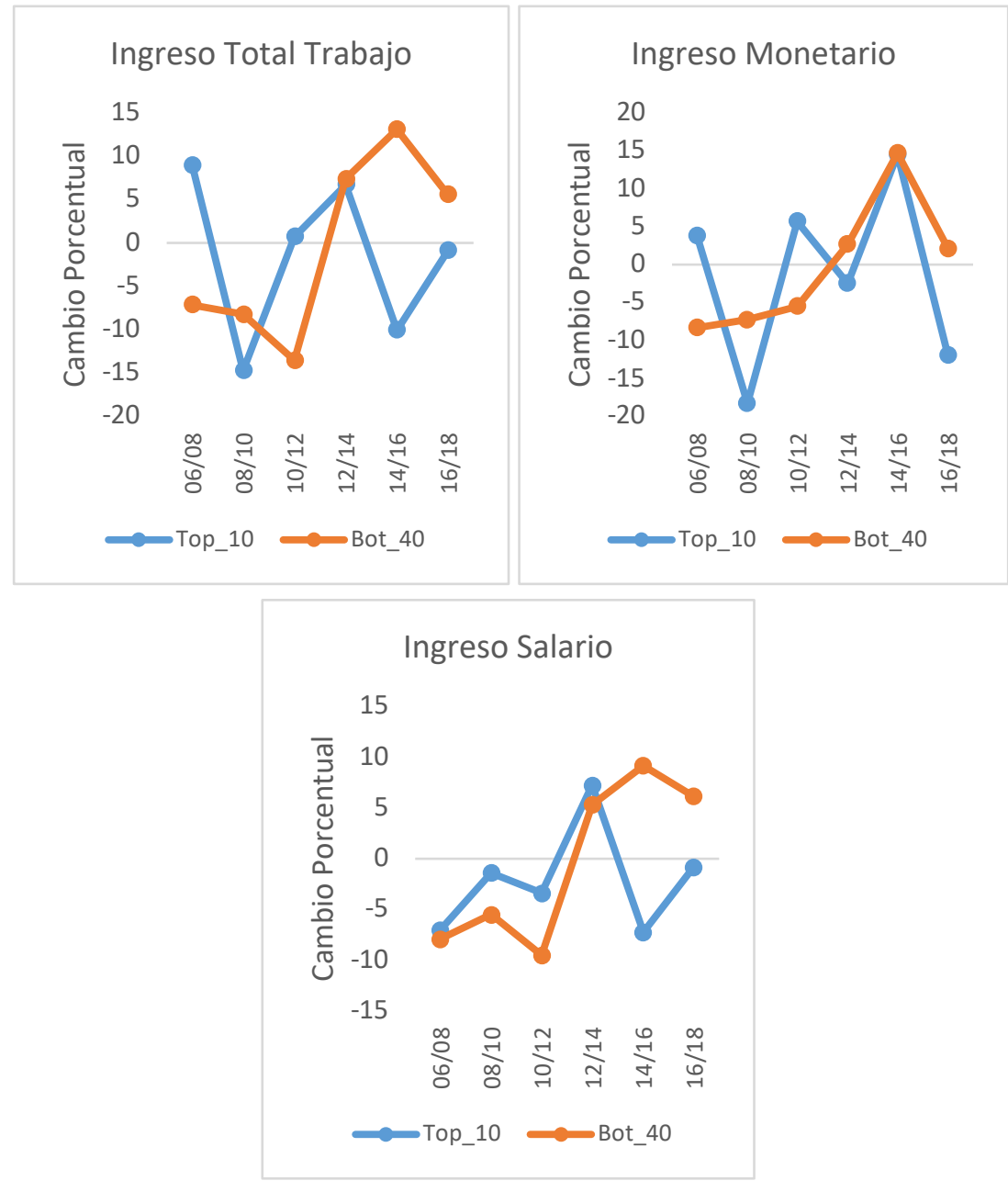

Fuente: Elaboración propia con información de la ENIGH, diferentes años.

En los párrafos anteriores mostramos evidencia que nos permite entender mejor la disminución de la desigualdad del ingreso monetario de los hogares mexicanos, durante el periodo de contracción económica causada por la Gran Recesión. Si bien los cambios en la distribución del ingreso dependen de lo que ocurra en todos los percentiles de la distribución de hogares, nuestro análisis indica que una de las principales razones de los cambios observados 
en la desigualdad del ingreso monetario es que hubo una mayor afectación en los ingresos de los hogares que se ubican en el Top_10 de la distribución, en relación con la afectación que tuvieron los hogares que se ubican en el Bot_40. Este hecho no indica que los ingresos de los hogares del Bot_40 no se vieran afectados por la crisis, sino más bien, que la afectación que tuvieron fue menor, debido a la redistribución que se dio por las transferencias de todo tipo. Sin embargo, cuando se observan los ingresos salariales, lo que observamos es un incremento en la desigualdad que logra ser revertido por otros componentes del ingreso de los hogares. Aun cuando las transferencias jugaron un papel importante para mitigar el impacto del choque negativo en los hogares con menores ingresos, fue principalmente la diferencia en el impacto que sufrieron los ingresos por negocios en los hogares, de los distintos grupos, lo que revierte el aumento de la desigualdad salarial. La gráfica 2 muestra que el impacto negativo de la Gran Recesión sobre los hogares mexicanos que se ubican en el Top_10 fue 2.5 veces el impacto sobre el Bot_ 40 .

\section{El impacto regional}

Con el fin de identificar si el impacto observado tiene un comportamiento regional, dividimos el país en 8 áreas, considerando la regionalización de Bassols Batalla (1994), que se basa en las características geográficas, históricas y económicas de las entidades federativas (tabla 6).

Tabla 6

Regiones y Entidades Federativas

\begin{tabular}{ll}
\hline Región & Estados \\
\hline Noroeste & $\begin{array}{l}\text { Baja California, Baja California Sur, Sonora, } \\
\text { Sinaloa, Nayarit }\end{array}$ \\
Norte & $\begin{array}{l}\text { Chihuahua, Coahuila, Durango, Zacatecas, San Luis } \\
\text { Potosí }\end{array}$ \\
Noreste & Nuevo León, Tamaulipas. Centro Occidente \\
Centro & Jalisco, Aguascalientes, Colima, Michoacán, \\
occidente & Guanajuato \\
Centro Sur & Querétaro, Estado de México, Distrito Federal, \\
& Morelos, Hidalgo, Tlaxcala, Puebla \\
Pacífico Sur & Guerrero, Oaxaca, Chiapas \\
Golfo de & Veracruz, Tabasco \\
México &
\end{tabular}




\section{Península de Campeche, Yucatán y Quintana Roo}

\section{Yucatán}

Fuente: Elaboración propia con información Bassols (1994).

Los cambios porcentuales en el índice de Gini 2008-2010 para las cuatro dimensiones del ingreso y las diferentes regiones, se muestran en la gráfica 3.

\section{Grafica 3}

Cambio porcentual del Índice de Gini y crecimiento del PIB 2008/2010 por región y dimensión ${ }^{5}$

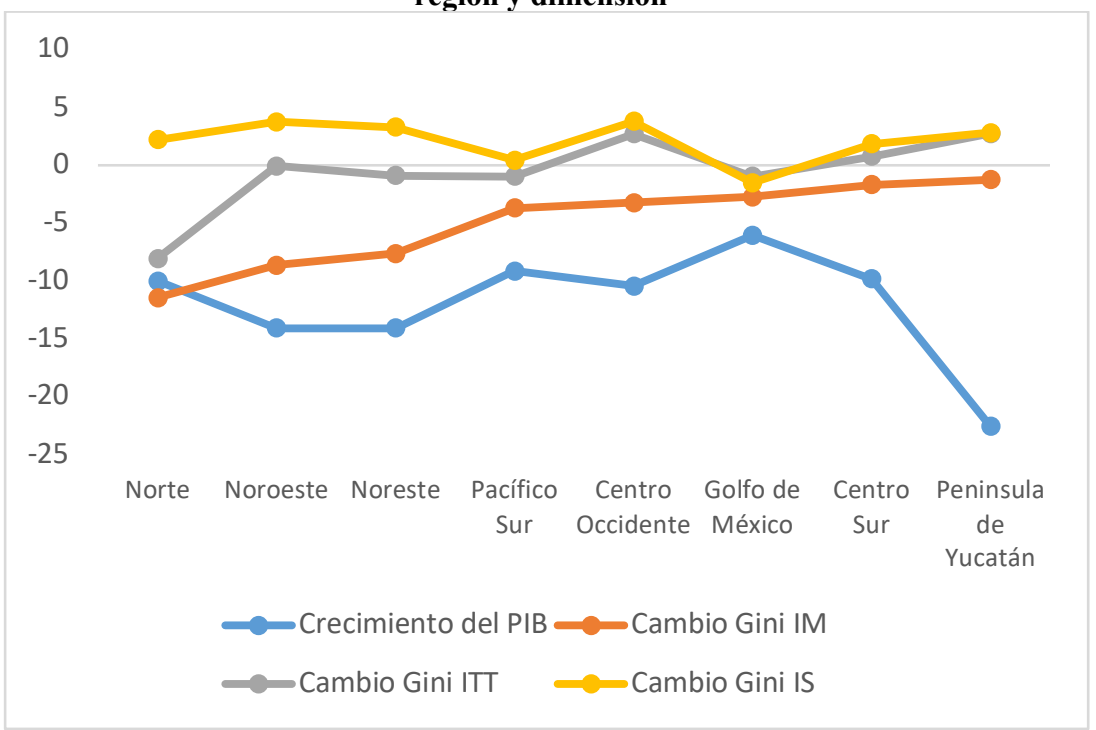

Fuente: Elaboración propia con información de le ENIGH, diferentes años.

Si bien en nivel nacional el índice de Gini del ingreso salarial aumentó, esto no ocurrió para todas las regiones. En el caso de la región del golfo de México, hubo una ligera disminución y para la región del pacífico sur el incremento fue muy pequeño. El resto de las regiones sufrieron incrementos en la desigualdad, principalmente en el centro occidente, en el noreste y noroeste. Al analizar los cambios del promedio del ingreso salarial para el Bot_40 y el Top_10, en cada región, podemos observar que existen diferencias importantes. Hay tres regiones en las que la caída del ingreso salarial para el Top_10 fue más fuerte que para el Bot 40, particularmente en el caso de la región del Golfo de México donde el promedio del Bot_40 prácticamente no cambió y el del Top_10, cayó $10.3 \%$ (ver gráfica 4 ). 
En el resto de las regiones, los ingresos salariales promedio del Bot_40 sufrieron un impacto negativo mayor que los del Top_10. Incluso en el caso del Centro Sur y del Noreste, los promedios del Top_10 tuvieron incrementos de $4.5 \%$ y $1.9 \%$ durante ese periodo, respectivamente; mientras que, en el caso de la Península de Yucatán, prácticamente no cambió.

Como puede verse en la gráfica 3 , al considerar los ingresos por negocios en la dimensión del ingreso total por trabajo, con excepción de la región del Golfo de México, el índice de Gini baja, o prácticamente no cambia, en comparación con el correspondiente al ingreso salarial, como es el caso de la Península de Yucatán. En esta última región, el promedio del ingreso total por trabajo para el Bot_40 y para el Top_10 caen en la misma proporción (gráfica 4), y dado que su coeficiente de Gini no cambió, en promedio, es probable que el ingreso por negocios haya caído en la misma proporción para el resto de los hogares. En el caso del Golfo, hay una caída de mayor proporción en el ingreso promedio para el Top_10 que para el Bot_40, sin embargo, los cambios en los índices de Gini para el IS y el ITT son de -1.5058 y -0.9589 , respectivamente. Es decir que, si bien en ambas dimensiones de ingreso hubo una disminución del coeficiente de Gini, en contraste con el resto de las regiones, la inclusión de los ingresos por negocios provoca una menor caída del coeficiente que cuando solo se consideran los ingresos salariales. Al igual que en estas dos regiones, la del Golfo y la de la Península de Yucatán, el ingreso total por trabajo, para el Top_10 tuvo una caída igual o mayor que para el Bot_40, comparada con en el caso de la región Noreste donde tuvo un aumento.

Como puede verse en la gráfica 4, existen diferencias importantes en las dinámicas del ingreso total por trabajo para estos dos grupos en las diferentes regiones. Al igual que en el Golfo y en la Península de Yucatán, en el resto de las regiones el ingreso total por trabajo para el Top_10 tuvo una caída igual o mayor que para el Bot_40, e incluso en el caso de la región Noreste, el ingreso promedio del Bot_40 tuvo un aumento. Las diferencias entre los dos grupos de hogares varían desde cero hasta 26.9 puntos porcentuales, en el caso de la región Norte.

En la gráfica 3, se puede observar como la desigualdad tiende a aumentar en las regiones donde el PIB se contrajo más. Este comportamiento, de alguna manera contracíclico, se observa en el caso de los ingresos salariales y de los ingresos totales por trabajo, que se encuentran vinculados con el pago a los factores de la producción. En el caso del ingreso monetario, esta relación se pierde dado que incluye principalmente componentes que no están ligados al pago de factores; en gran medida, las transferencias buscan mitigar los impactos negativos en el ingreso de los hogares y, además, el ingreso por rentas y pagos de activos es probablemente uno de los rubros que presenta mayor subregistro en la encuesta. Aunque sigue habiendo diferencias regionales 
importantes en el cambio del coeficiente de Gini, en el caso del ingreso monetario, en todas las regiones se registró una disminución de su valor (gráfica 3). De igual forma, la heterogeneidad en las diferencias en los cambios del ingreso monetario para el Bot_40 y el Top_10 es muy grande; sin embargo, en este caso, en todas las regiones el ingreso monetario promedio disminuyó tanto para el Bot_40 como para el Top_10, y este último siempre tuvo una mayor caída que el primero (gráfica 4).

\section{Gráfica 4}

Cambio porcentual en el ingreso promedio 2008/2010 por región y dimensión 

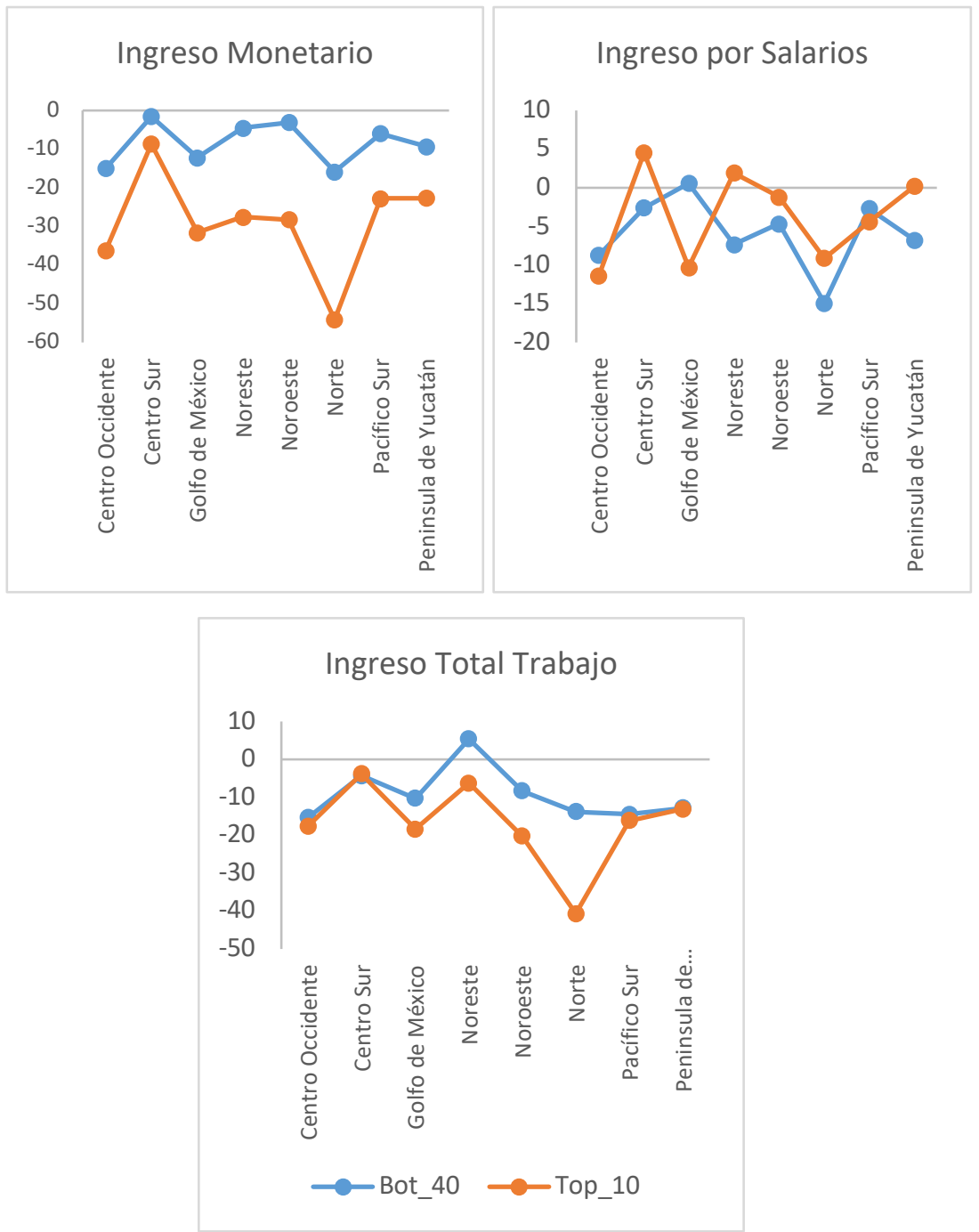

Fuente: Elaboración propia con información de le ENIGH, diferentes años.

Hasta aquí, hemos asumido que los cambios descritos en los ingresos promedio del Top_10 y del Bot_40 en el nivel regional, responden al choque negativo que implicó la Gran Recesión, por lo que a continuación presentamos un análisis econométrico para validar la significancia estadística sobre el mayor impacto de la Gran Recesión en el Top_10, en relación con el Bot_40.

Las diferencias en la dinámica de cada región están ligadas a la composición sectorial de su producción y a su peso en el comercio exterior del país. Sin embargo, aun regiones que pudieran ser similares en ese aspecto, como las del 
norte o las del centro, presentan dinámicas muy distintas, tendrían que analizarse con más detalle.

\section{Análisis econométrico}

Para validar estadísticamente los impactos que hemos descrito en este trabajo, utilizamos un modelo de regresión lineal con métodos robustos, para hacer un análisis de diferencias en diferencias. Para ello utilizamos las ENIGH del 2008 y del 2010 y, de cada una de ellas, obtenemos los hogares que se ubican en el Top_10 y aquellos que están en el Bot_40. En nuestra regresión, la variable a explicar es el ingreso monetario de los hogares (IM). Utilizamos cuatro grupos de variables de control. El primero incluye factores sociodemográficos del hogar, como lo son: la educación, la edad, la edad al cuadrado, el sexo del jefe de familia, la situación conyugal y la relación entre mayores y menores que habitan en el hogar. En el segundo grupo identificamos las variables económicas, en las que incluimos las horas trabajadas. En el tercer grupo capturamos los efectos regionales con una variable dicotómica para cada región, que toma el valor 1 para la región de referencia y cero para cualquier otra región. Tomamos como referencia la región 1 .

Finalmente, en un cuarto grupo incluimos tres variables dicotómicas para determinar si la Gran Recesión tuvo algún impacto sobre el ingreso. Una de ellas toma el valor 1, si el hogar corresponde al Top_10 y cero si se encuentra en el Bot_40. Una segunda variable dicotómica toma el valor 1 para los datos del año 2010 , fecha en la que se manifiestan los efectos de la Gran Recesión sobre los hogares, y cero en otro caso. Finalmente, incluimos una última variable de interacción entre las dos anteriores. La muestra total para el modelo es de 28,795 hogares.

El modelo econométrico tiene la siguiente forma:

$\ln I M=\beta_{0}+\beta_{1} \boldsymbol{R}+\beta_{2} \boldsymbol{H}+\beta_{3} \mathbf{X}+\beta_{4} \boldsymbol{D}$

donde $\beta_{1}$ es un vector con siete entradas, correspondientes a cada región con excepción de la región de referencia; $\beta_{2}$ es un vector con un único elemento; $\beta_{3}$ es un vector de siete parámetros y $\beta_{4}$ contiene tres parámetros. La descripción de nuestras variables se presenta en la tabla 7.

El coeficiente $\beta_{4,1}$ tendrá el impacto de la crisis sobre el ingreso de los hogares del Bot_40, mientras que la suma de los coeficientes $\beta_{4,2}$ y $\beta_{4,3}$ representará el impacto sobre los hogares del Top_10. Los parámetros en el vector $\beta_{1}$ definen las diferencias de ingreso entre regiones. El resto de los parámetros corresponden a variables sociodemográficas de control. Nuestra variable dependiente se encuentra en logaritmos, por lo cual los coeficientes representan semielasticidades. 
Tabla 7

Descripción de Variables

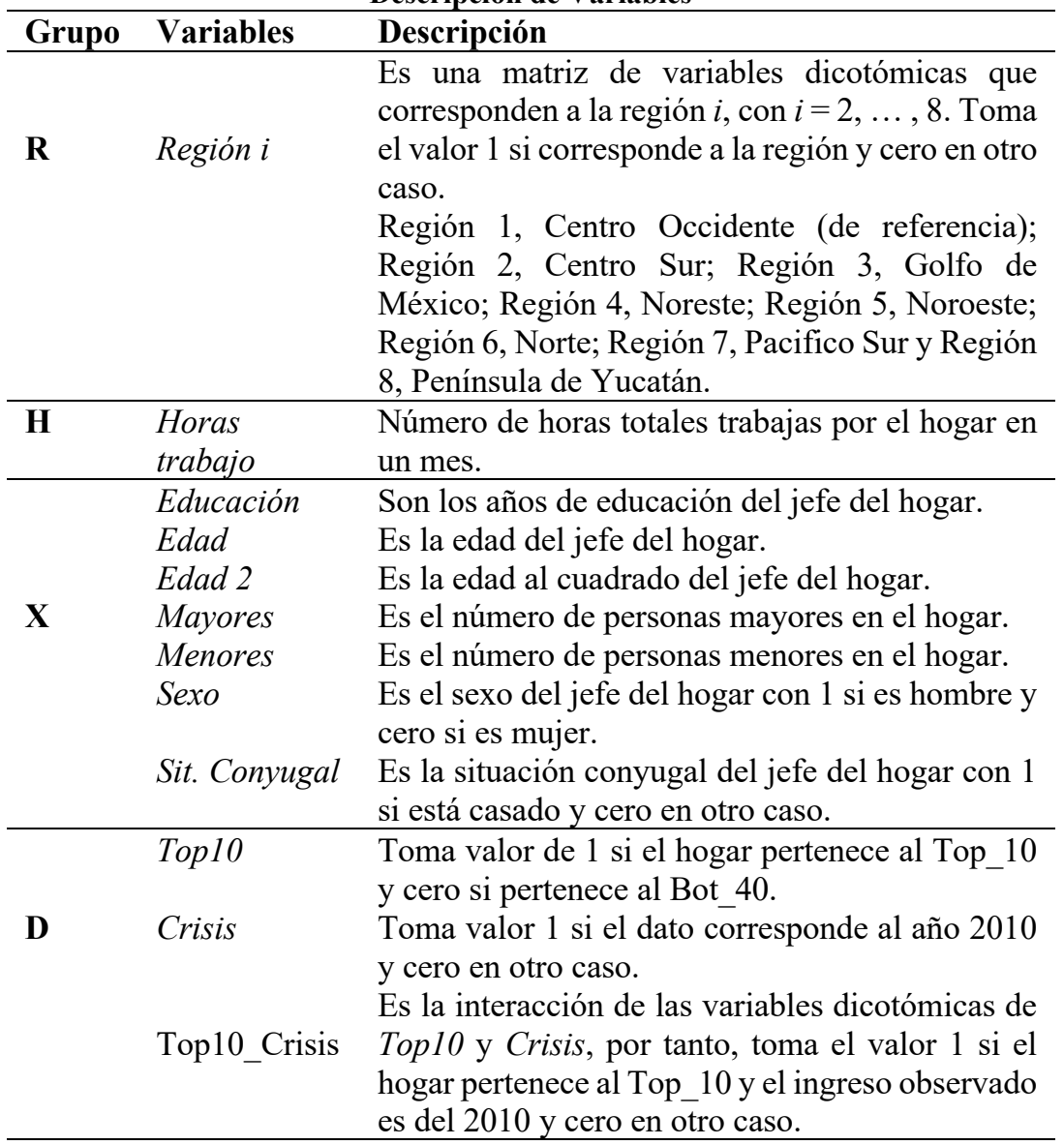

Tabla 8

Estimaciones del modelo (MCO método robusto)

Variable Dependiente:

In_ingreso

$\begin{array}{llll}\text { Constante } & 7.794817^{* * *} & \text { Edad } & 0.001790 * * *\end{array}$




\begin{tabular}{|c|c|c|c|}
\hline & $(0.00110)$ & & $(0.000043)$ \\
\hline \multirow[t]{2}{*}{ Región 2} & $0.014107 * * *$ & Edad 2 & $-0.000035 * * *$ \\
\hline & (0.00033) & & $(0.0000004)$ \\
\hline \multirow[t]{2}{*}{ Región 3} & $-0.102755^{* * *}$ & Mayores & $0.049227^{* * *}$ \\
\hline & $(0.00042)$ & & $(0.00010)$ \\
\hline \multirow[t]{2}{*}{ Región 4} & $0.053167 * * *$ & Menores & $0.000454 * * *$ \\
\hline & $(0.00046)$ & & $(0.00010)$ \\
\hline \multirow[t]{2}{*}{ Región 5} & $0.030671 * * *$ & Sexo & $-0.031495 * * *$ \\
\hline & $(0.00045)$ & & $(0.00036)$ \\
\hline \multirow[t]{2}{*}{ Región 6} & $-0.015453^{* * *}$ & Sit. conyugal & $0.094532 * * *$ \\
\hline & $(0.00043)$ & & $(0.00037)$ \\
\hline \multirow[t]{2}{*}{ Región 7} & $-0.254445^{* * *}$ & Top10 & $2.140118^{* * *}$ \\
\hline & $(0.00043)$ & & $(0.00043)$ \\
\hline \multirow[t]{2}{*}{ Región 8} & $-0.024525 * * *$ & Crisis & $-0.085464 * * *$ \\
\hline & $(0.00063)$ & & $(0.00063)$ \\
\hline Horas & $0.002061 * * *$ & Top10_Crisis & $-0.096542 * * *$ \\
\hline trabajo & $(0.000002)$ & & $(0.000466)$ \\
\hline \multirow[t]{2}{*}{ Educación } & $0.03162 * * *$ & & \\
\hline & $(0.000029)$ & & \\
\hline
\end{tabular}

Núm. de obs. 28526657

$\mathrm{R}^{2} 0.7638$

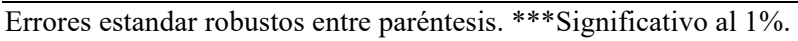

Los resultados del modelo de regresión se muestran en la tabla 8. Todas las variables de control conservan el signo esperado y resultaron significativas en un $99 \%$. El ingreso de los hogares tiene un comportamiento de "U" invertida con respecto a la edad, y aumenta con la educación y con las horas trabajadas. La presencia de personas mayores y de niños en el hogar tiene un efecto positivo sobre el ingreso del hogar, al igual que un estado civil de casado para el jefe de familia. Todas las diferencias regionales fueron significativas.

Los coeficientes de interés, $\beta_{4,2}$ y $\beta_{4,3}$, son significativos y guardan el signo esperado, lo cual respalda la hipótesis del impacto de la Gran Recesión en la disminución de la desigualdad del ingreso monetario. De acuerdo con estos resultados, la Gran Recesión habría reducido el ingreso monetario de los 
hogares del Bot_40 en 8.5\%, mientras que el impacto en al caso del Top_10 fue de casi el doble con $18.21 \%$.

Estos resultados nos permiten validar estadísticamente lo que se ha discutido en la sección anterior, donde mostramos que, en el corto plazo, la Gran Recesión provocó una disminución a la desigualdad del ingreso monetario, ocasionada principalmente por un mayor impacto negativo en los hogares del Top_10, respecto al Bot_40, mientras que, para el ingreso total por trabajo, la disminución de los indicadores de desigualdad fue mucho menor, y para el ingreso salarial, hubo un aumento.

\section{Conclusiones}

A partir de la Gran Recesión, se ha publicado un número importante de trabajos enfocados en analizar las consecuencias que la crisis tuvo sobre la distribución del ingreso. Sin embargo, para el caso de México, no se han analizado con suficiente profundidad los cambios que la contracción económica produjo en la distribución del ingreso entre los diferentes sectores de la población.

Con este trabajo, contribuimos a la literatura enfocada en este tema, a través del análisis de la dinámica de diferentes dimensiones del ingreso de los hogares. La dimensión más simple que analizamos es el ingreso por trabajo asalariado. La segunda es el ingreso total por trabajo, que incluye el ingreso por salarios, más el ingreso por negocios propios y autoempleo. La última dimensión agrega al ingreso total por trabajo los ingresos por rentas y pagos de activos, más transferencias, que incluyen pensiones y jubilaciones, transferencias de gobierno y entre hogares, y remesas.

Nuestro análisis es consistente con aquellos que registran una caída de la desigualdad del ingreso corriente de los hogares, durante el periodo de contracción económica, causada por la Gran Recesión (Cortés, 2013; Cortés y Vargas, 2017; Iniguez-Montiel y Kurosaki, 2018); pero, también mostramos evidencia que sugiere que la caída de la desigualdad de nuestra categoría de ingreso monetario, fue resultado de los efectos del choque negativo sobre los ingresos por negocios y autoempleo, y sobre todo de las transferencias entre hogares, las pensiones y las transferencias gubernamentales. Durante el periodo de 2008-2010 en el que se registra la caída del PIB como resultado de la Gran Recesión; a nivel nacional, los ingresos de los hogares en sus tres dimensiones cayeron, en promedio, tanto para aquellos en los deciles bajos como para los del decil más alto.

En contraste con el ingreso monetario, la desigualdad del ingreso salarial aumentó en ese periodo. Sin embargo, la contracción económica afectó en mayor proporción el ingreso por negocios y autoempleo de los hogares en los 
deciles altos, que al de los hogares en los deciles bajos. Este efecto fue lo suficientemente fuerte como para revertir la tendencia de la desigualdad del ingreso salarial, por lo que la desigualdad con respecto al ingreso total por trabajo disminuyó.

Hay que considerar que es en el ingreso por negocios propios y autoempleo, donde más se registran los ingresos del trabajo informal, por lo que es muy probable que el empleo informal juegue un papel importante en la caída de los indicadores de desigualdad, durante el periodo de contracción.

Finalmente, la caída en las rentas, que afectó más a los hogares con mayores ingresos, también fue importante en la disminución de la desigualdad del ingreso monetario, pero el componente del ingreso monetario que magnifica la caída de los indicadores de desigualdad que ya se observaba en el ingreso total del trabajo, fueron las transferencias. Este fenómeno puede considerarse, como lo que se ha denominado: "estancamiento que favorece a los pobres" (Ramírez y Valdez, 2018), o disminución de desigualdad por empobrecimiento (Cortés, 2011), que en este caso ocurre a la mayor afectación que sufrieron los ingresos por negocios y autoempleo en los deciles superiores, pero que no se observa en la desigualdad de los ingresos por trabajo asalariado.

En el nivel regional, el comportamiento de las tres dimensiones de ingreso no es homogéneo. Cada región tiene una dinámica propia que puede diferir del comportamiento promedio en el nivel nacional. Si bien existe una relación negativa entre el crecimiento del PIB en cada región y el cambio de los coeficientes de Gini para el ingreso salarial y el ingreso total por trabajo, lo cual es consistente con un comportamiento contracíclico de la desigualdad, la contracción económica impactó de forma distinta estas dimensiones del ingreso en las diferentes zonas del país. No en todas las regiones hubo una mayor caída de los ingresos salariales para los hogares en los deciles bajos. De igual manera, hay regiones donde la afectación a los ingresos por negocios no fue más fuerte en los hogares con mayores ingresos. En términos de política económica y política pública, estas diferencias son relevantes. Las distintas dinámicas que se observan en el nivel regional deben ser analizadas con mayor detenimiento al diseñar programas y ejecutar cualquier política redistributiva, que tenga como objetivo disminuir la desigualdad y fortalecer la capacidad de los hogares, para enfrentar los impactos de choques negativos de la economía.

\section{Referencias}


[1] Acemoglu, D. (2011, January). Thoughts on inequality and the financial crisis. In AEA meeting, Denver (Vol. 7).

[2] Alvaredo, F., y Gasparini, L. (2015). Recent trends in inequality and poverty in developing countries. Handbook of income distribution, 2, 697-805.

[3] Atkinson, A. B., y Brandolini, A. (2006). From earnings dispersion to income inequality.

[4] Barlevy, G., y Tsiddon, D. (2006). Earnings inequality and the business cycle. European Economic Review, 50(1), 55-89.

[5] Bassols Batalla, Á. (1994). Regiones para el desarrollo de México. Problemas del Desarrollo. Revista Latinoamericana de Economía, 25(96).

[6] Bogliacino, F y Maestri, V. (2016) Wealth inequality and the Great Recession, Intereconomics: Review of European Economic Policy, 51(2), 61-66.

[7] Brzezinski, M. (2018). Income inequality and the Great Recession in Central and Eastern Europe. Economic Systems, 42(2), 219-247.

[8] Camacho, M., y Palmieri, G. (2019). Do economic recessions cause inequality to rise? Journal of Applied Economics, 22(1), 304-320.

[9] Camberos, M., y Bracamontes, J. (2015). Las crisis económicas y sus efectos en el mercado de trabajo, en la desigualdad y en la pobreza de México. Contaduría y administración, 60, 219-249.

[10] Carmona, N., y Caamal C. G. (2018). ¿Las transferencias federales han logrado reducir la desigualdad en las entidades federativas en México? EconoQuantum, 15(1), 31-51.

[11] Cortés, F. (2011). Desigualdad económica y poder en México. CEPAL. Recuperado de https://repositorio.cepal.org/handle/11362/24260

[12] Cortés, F. (2012). Desigualdad económica en México: Enfoques conceptuales y tendencias empíricas. Estudios Sociológicos, 157-189.

[13] Cortés, F. (2013). Medio siglo de desigualdad en el ingreso en México. Economía UNAM, 10(29), 12-34.

[14] Cortés, F y Vargas, D. (2017). La evolución de la desigualdad en México: viejos y nuevos resultados. Revista de Economía Mexicana (2), 39-96.

[15] Chiquiar, D. (2008). Globalization, regional wage differentials and the StolperSamuelson Theorem: Evidence from Mexico. Journal of International Economics, 74(1), 70-93.

[16] De Ferranti, D., Perry, G. E., Ferreira, F., y Walton, M. (2004). Inequality in Latin America: breaking with history?. The World Bank.

[17] De la Torre, A., Yeyati, E., Beylis, G., Didier, T., Rodríguez-Castelán, C., y Schmukler, S. (2014). Inequality in a lower growth Latin America. Latin America and the Caribbean Semiannual Report (October). Washington, DC: World Bank.

[18] De la Torre, A., Messina, J., \& Silva, J. (2017). The inequality story in Latin America and the Caribbean: Searching for an explanation. In Has Latin American inequality changed direction? 317-338. Springer, Cham.

[19] Del Castillo-Negrete, M. (2015). La magnitud de la desigualdad en el ingreso y la riqueza en México. Una propuesta de cálculo. Serie Estudios y Perspectivas. Sede Subregional de la CEPAL en México, Naciones Unidas, México, D.F

[20] Dimelis, S., y Livada, A. (1999). Inequality and business cycles in the US and European Union countries. International Advances in Economic Research, 5(3), 321-338. 
[21] Domínguez, M. I. D., y García, B. G. (2016). Income Distribution and the 20082012 Economic Crisis: The Latin American Experience. Selected Papers from the Asia Conference on Economics y Business Research 2015, 121126.

[22] Emmons, W. R., y Noeth, B. J. (2012). Household financial stability: Who suffered the most from the crisis? The Regional Economist, 20(4),11-17

[23] Espinal, A. E. (2010). Gran Recesión 2008-2009 en EE. UU. y México: Un enfoque regional. Paradigma económico, 2(2), 5-40.

[24] Esquivel, G., y Cruces, G. (2011). The dynamics of income inequality in Mexico since NAFTA [with Comment]. Economía, 12(1), 155-188.

[25] Esquivel, G., y Messmacher, M. (2002). Sources of regional (non) convergence in Mexico. El Colegio de México y Banco de Mexico, Mexico City.

[26] Gasparini, L., y Lustig, N. (2011). The Rise and Fall of Income Inequality in Latin America in Ocampo, José Antonio y Ros, Jaime (Eds.), The Oxford Handbook of Latin American Economics. Oxford University Press. pp 691714.

[27] González, J. Z., Pérez, J. F. M., y Montoya, F. R. (2009). La crisis financiera y económica del 2008. Origen y consecuencias en los Estados Unidos y México. El cotidiano, 157, 17-27.

[28] Gutiérrez Flores, L. (2008). La distribución del ingreso en México: un análisis regional, 1990-2004. Problemas del desarrollo, 39(152), 139-163.

[29] Grabka, M. (2015). Income and wealth inequality after the financial crisis: The case of Germany. Empirica, 42, 371-390. https://doi.org/10.1007/s10663-0159280-8

[30] Iniguez-Montiel, A. J., \& Kurosaki, T. (2018). Growth, inequality and poverty dynamics in Mexico. Latin American Economic Review, 27(1), 1-25.

[31] Jenkins, S. P., Brandolini, A., Micklewright, J., Nolan, B., y Basso, G. (2013-A). The Great Recession and its consequences for household incomes in 21 countries. In Jenkins, S. P., Brandolini, A., Micklewright, J., y Nolan, B. (Eds.). (2012). The great recession and the distribution of household income (33-89). OUP Oxford.

[32] Jenkins, S. P., Brandolini, J., Micklewright, J., y Nolan, B. (2013-B). Scope, review of approaches, and evidence from the past. In Jenkins, S. P., Brandolini, A., Micklewright, J., \& Nolan, B. (Eds.). (2013). The great recession and the distribution of household income (1-32). OUP Oxford,

[33] Krozer, A. (2015). The Inequality We Want: How Much Is Too Much? Journal of International Commerce, Economics and Policy, 06(03), 1550016. https://doi.org/10.1142/S1793993315500167

[34] López-Calva, L. F., y Lustig, N. C. (Eds.). (2010). Declining inequality in Latin America: A decade of progress?. Brookings Institution Press.

[35] Lustig, N. (2010) "El impacto de 25 años de reformas sobre la pobreza y la desigualdad.”, en Nora Lustig (coord.). Los Grandes problemas de México. Vol. IX. Crecimiento Económico y Equidad. El Colegio de México.

[36] Lustig, N., Lopez-Calva, L. F., y Ortiz-Juarez, E. (2013). Declining inequality in Latin America in the 2000s: The cases of Argentina, Brazil, and Mexico. World development, 44, 129-141.

[37] Maliar, L., Maliar, S., y Mora, J. (2005). Income and wealth distributions along the business cycle: Implications from the neoclassical growth model. The $B E$ Journal of Macroeconomics, 5(1). 
[38] Meyer, B. D., y Sullivan, J. X. (2013). Consumption and income inequality and the great recession. American Economic Review, 103(3), 178-83.

[39] Morelli, S., y Atkinson, A. B. (2015). Inequality and crises revisited. Economia Politica, 32(1), 31-51.

[40] Muriel, A., y Sibieta, L. (2009). Living standards during previous recessions. Institute for Fiscal Studies (IFS), Briefing Note no. 85 (http://www.ifs.org.uk/bns/bn85.pdf).

[41] Palma, J. G. (2011). Homogeneous middles vs. Heterogeneous tails, and the end of the 'inverted-U': It's all about the share of the rich. Development and Change, 42(1), 87-153.

[42] Parker, S. C. (1998). Income inequality and the business cycle: A survey of the evidence and some new results. Journal of Post Keynesian Economics, 21(2), 201-225.

[43] Parker, J. A., y Vissing-Jorgensen, A. (2009). Who bears aggregate fluctuations and how? American Economic Review, 99(2), 399-405.

[44] Parker, J. A., y Vissing-Jorgensen, A. (2010). The increase in income cyclicality of high-income households and its relation to the rise in top income shares (No. w16577). National Bureau of Economic Research.

[45] Perri, F., y Steinberg, J. (2012). Inequality and redistribution during the Great Recession. Federal Bank of Minneapolis, Economic Policy Paper 12-1.

[46] Pfeffer, F. T., Danziger, S., y Schoeni, R. F. (2013). Wealth disparities before and after the Great Recession. The ANNALS of the American Academy of Political and Social Science, 650(1), 98-123.

[47] Piketty, T., y Saez, E. (2003). Income inequality in the United States, 19131998. The Quarterly journal of economics, 118(1), 1-41.

[48] Piketty, T. (2014). El capital en el siglo XXI. Fondo de cultura económica.

[49] Piketty, T., y Saez, E. (2013). Top incomes and the great recession: Recent evolutions and policy implications. IMF economic review, 61(3), 456-478.

[50] Piketty, T., y Zucman, G. (2014). Capital is back: Wealth-income ratios in rich countries 1700-2010. The Quarterly Journal of Economics, 129(3), 12551310.

[51] Ramírez, B., y Valdez, X. (2018). Pobreza, desigualdad y crecimiento económico: Una estimación para las regiones en México. En México en el umbral del siglo XXI: hacia un entendimiento de sus principales problemas económicosociales (93-114). Universidad de Guadalajara.

[52] Reinhart, C. M., y Rogoff, K. S. (2012). Esta vez es distinto: ocho siglos de necedad financiera. Fondo de Cultura Económica.

[53] Rivas, M. G. (2007). The effects of trade openness on regional inequality in Mexico. The Annals of Regional Science, 41(3), 545-561.

[54] U.S. Congress (2010) Income Inequality and the Great Recession, Report del U.S. Congress Joint Economic Committee. (https://www.jec.senate.gov/public/_cache/files/91975589-257c-403b-80938f3b584a088c/income-inequality-brief-fall-2010-cmb-and-ces.pdf).

[55] Székely, M. (2005). Pobreza y desigualdad en México entre 1950 y 2004. El Trimestre Económico, 72(288), 913-931.

[56] Stiglitz, J. (2009). Joseph Stiglitz and why inequality is at the root of the recession. Next left website, 9 January.

[57] Wolff, E. N. (2013). The asset price meltdown, rising leverage, and the wealth of the middle class. Journal of Economic Issues, 47(2), 333-342. 
